\title{
Neural Representations of Gain-Loss Frequency in Older and Younger Adults
}

Hilary J. Don ${ }^{1}$, Tyler Davis ${ }^{2}$, Kimberly L. Ray ${ }^{3}$, Megan C McMahon ${ }^{3}$, Astin C. Cornwall ${ }^{1}$, David M. Schnyer ${ }^{3}, \&$ Darrell A. Worthy ${ }^{1}$

\author{
${ }^{1}$ Texas A\&M University \\ ${ }^{2}$ Texas Tech University \\ ${ }^{3}$ University of Texas at Austin
}

Correspondence concerning this article should be addressed to Hilary J. Don, who is now at Division of Psychology and Language Sciences University College London 26 Bedford Way, Bloomsbury, London WC1H 0AP, United Kingdom h.don@ucl.ac.uk 


\section{Abstract}

2 Research on the biological basis of reinforcement-learning has focused on how brain regions

3 track expected value based on average reward. However, recent work suggests that humans are

4 more attuned to reward frequency. Furthermore, older adults are less likely to use expected

5 values to guide choice than younger adults. This raises the question of whether brain regions

6 assumed to be sensitive to average reward, like the medial and lateral PFC, also track reward

7 frequency, and whether there are age-based differences. We scanned older and younger adults

8 performing the Soochow Gambling task, which separates reward frequency from average

9 reward. Overall, participants preferred options that provided negative net payoffs, but frequent

10 gains. Older adults improved less over time, were more reactive to recent negative outcomes, and

11 showed greater frequency-related activation in several regions, including lateral PFC. We also

12 found broader recruitment of prefrontal and parietal regions in older adults, which may indicate

13 compensation.

14 Keywords: decision-making; expected value; aging; compensation; model-based FMRI 


\section{Introduction}

Decisions in every-day life often involve choosing amongst multiple options that each have potential for positive or negative outcomes. For example, deciding which stocks to invest in, which school to send your children to, or which property to buy all come with the possibility of good and bad outcomes. Making optimal choices is assumed by many researchers to require an estimation and comparison of the expected value (EV) of alternative choice options (Edwards, 1954; Rangel et al., 2008; Samanez-Larkin and Knutson, 2015). Most prominent models of reinforcement learning assume that $\mathrm{EV}$ is based on the average reward provided by each option (e.g., delta models; Rescorla \& Wagner, 1972; Widrow \& Hoff, 1960; Williams, 1992). Several studies have found that, compared to younger adults, older adults are less sensitive to differences in EV, or less reliant on EV to make decisions (Brand \& Markowitsch, 2010; Brand \& Schiebener, 2013; Deakin, Aitken, Robbins, \& Sahakian, 2004, Weller et al., 2011). For example, Weller, King, Figner and Denburg (2019) found that older adults were less likely to base decisions on expected value than younger adults in a risky decision-making task. Older adults used only a subset of information, adapting their choices based on probability information (presented as frequencies of losses), but not on the magnitude of gains and losses.

These declines in value-based decision-making performance appear to be due to changes in executive function and working memory (Li et al., 2001; Salthouse, 2004), changes in associated cognitive control regions in the lateral PFC (Braver et al., 2001; Sharp, Scott, Mehta, \& Wise, 2006) as well as structural declines in striatal regions (Bäckman et al., 2006, Li et al., 2001) that are associated with processing reward (Hare et al., 2008; Pagnoni et al., 2002; Chowdhury et al., 2013). Age related changes may also be present in orbitofrontal cortex (OFC; 
1 Resnick, Lamar, \& Driscoll, 2007), and the lateral OFC has been implicated in suppression of

2 previously rewarded responses (Elliott, Dolan, \& Frith, 2000).

Extensive work on the role of prefrontal and striatal brain regions in value-based choice

4

5 has focused on how these regions track average reward using predictions from delta-like reinforcement learning models (Blair et al., 2006; Daw et al., 2006; Elliot, Dolan \& Frith, 2000; Hare et al, 2008; Pagnoni et al., 2002; Pessiglione et al., 2006). However, there are many contexts in which people use sources of information other than average reward to inform their expectations (Estes, 1976; Einhorn \& Hogarth, 1981). For example, people are often sensitive to the relative frequency of positive versus negative outcomes (e.g., Pang, Blanco, Maddox \& Worthy, 2017). People also prefer choice options that have been frequently rewarded, even if they have lower average reward than an alternative (Don, Otto, Cornwall, Davis \& Worthy, 2019). This result highlights the need to potentially re-evaluate the neural architecture of valuebased decision making - how they track reward frequency versus average reward - and how people's ability to use these aspects of reward history in decision making change as a function of healthy aging.

One task that neatly dissociates average reward from gain-loss frequency, and thus can be useful for isolating these computations using neuroimaging, is the Soochow Gambling Task (SGT; Chiu et al., 2008). The SGT is an experience-based decision-making task in which participants choose between four decks of cards that each have different schedules of earning and losing points, where the goal is to maximize rewards received. The reward schedule for each deck is shown in Table 1. Decks A and B are "bad decks" according to average reward, as they lead to long-term negative payoffs (-500 over 10 card draws). Decks C and D are "good decks", as they lead to long-term positive payoffs (+500 over 10 card draws). However, the bad decks 
1 provide small gains on $80 \%$ of trials, and large losses on $20 \%$ of trials, while the good decks

2 provide small losses on $80 \%$ of trials, and large gains on $20 \%$ of trials. Optimal performance in

3 this task therefore relies on participants using long-term payoffs to guide their choices. Healthy

4 participants typically perform poorly in this task, continuing to prefer the bad decks A and B,

5 suggesting their decisions are guided more by gain-loss frequency than average reward (Chiu et

6 al, 2008; Lin et al, 2009). This is further supported by work demonstrating that reinforcement

7 learning models provide better fits to Iowa Gambling Task (IGT; Bechara et al., 1994; 1997) and

8 SGT data when using decay-reinforcement updating rules, which base expected value on reward

9 frequency, than delta-reinforcement updating rules, which base expected value on average

10 reward (Ahn et al., 2008; Dai et al., 2015).

The SGT may be useful for re-evaluating how frequency and average reward information

12 are represented in regions of the brain associated with value-based decision making. For

13 example, although the ventral striatum is known to track average reward, and prediction errors

14 from average reward, it is less certain if and whether reward frequency information may affect

15 value representations. Likewise, the lateral PFC is known to track uncertainty related to outcome

16 variability (Kahnt, Heinzle, Park, \& Haynes, 2011; Schonberg, Fox, \& Poldrack, 2011), is

17 involved in state change uncertainty signals (Worthy et al., 2016), and tracks individual

18 differences in risk aversion (Christopoulos, Tobler, Bossaerts, Dolan, \& Schultz, 2009). On a

19 mechanistic level, the lateral PFC is thought to be involved in resolving conflicting information

20 (e.g., outcome variability) in cases of higher uncertainty, due to its more general role as a center

21 for cognitive control (Koechlin, Ody, and Kouneiher, 2003; Badre and D'esposito, 2009;

22 Breukelaar et al., 2017). Finally, OFC is involved in controlling and suppressing responses to

23 previous outcomes. Whether such control processes are primarily associated with average reward 
1 or reward frequency remains an open question. Reward frequency tends to do a better job of

2 predicting SGT behavior than average reward, and lateral PFC should thus be sensitive to

3 people's use of frequency in decisions. Choices with lower frequency value (i.e., the good decks

4 that yield losses more frequently) should also be associated with more conflict as they go against

5 participants' preferences for more frequent reward, and thus we'd expect that lateral PFC would

6 be negatively associated with frequency value.

In addition to the issue of reward frequency versus average reward, there is evidence that

8 older adults are more responsive to recent events (Besedes, Deck, Sarangi, \& Shor, 2012; Castel,

9 Rossi, \& McGillivray, 2012), particularly recent negative events. Older adults are more likely to

10 switch choices following large losses or negative prediction errors than younger adults (Worthy

11 et al., 2015; Worthy et al., 2016). They are also more likely to use a "win-stay-lose-shift"

12 (WSLS) heuristic, in which choices are based only on the outcome of the previous trial. That is,

13 choices are repeated if they provided reward on a previous trial, and switched if they were

14 unrewarded (Worthy \& Maddox 2012; Worthy, Otto \& Maddox, 2012). In comparison, younger

15 adults are more likely to use a recency-weighted average of past rewards to guide choices

16 (Worthy \& Maddox 2012; Worthy, Otto \& Maddox, 2012). Older adults are also more likely to

17 use reactive, rather than proactive cognitive control (Karayanidis, Whitson, Heathcote \& Michie,

18 2011). The use of simpler, reactive decision making strategies in older adults may compensate

19 for deficits in sensitivity to long-term average reward. However, the precise neural and

20 computational mechanisms associated with age-related differences in decision-making are still

21 unclear. 
Table 1

Reward schedule for 10 trials of the Soochow Gambling Task.

\begin{tabular}{lllll}
\hline & \multicolumn{2}{c}{ Bad decks } & \multicolumn{2}{c}{ Good decks } \\
\hline Draw from deck & Deck A & Deck B & Deck C & Deck D \\
\hline 1 & 200 & 100 & -200 & -100 \\
2 & 200 & 100 & -200 & -100 \\
3 & 200 & 100 & -200 & -100 \\
4 & 200 & 100 & -200 & -100 \\
5 & -1050 & -650 & 1050 & 650 \\
6 & 200 & 100 & -200 & -100 \\
7 & 200 & 100 & -200 & -100 \\
8 & 200 & 100 & -200 & -100 \\
9 & 200 & 100 & -200 & -100 \\
10 & -1050 & -650 & 1050 & 650 \\
\hline Net payoff & -500 & -500 & 500 & 500 \\
\hline
\end{tabular}

1

Several studies have found evidence of increased activation in lateral and inferior prefrontal brain regions for older adults relative to younger adults in a variety of cognitive tasks. These prefrontal regions are often associated with executive function, and can be recruited adaptively to meet task demands. An increase in activation in these regions is suggested to compensate for age-related neural decline that may otherwise affect task performance (Cabeza, 2002; Cabeza et al, 2002; 2004; Cappell et al., 2010; Park \& Reuter-Lorenz, 2009; Phillips \& Andres, 2010; Reuter-Lorenz \& Cappell, 2008; Reuter-Lorenz et al, 2000). Such compensatory activation should therefore be associated with improvement in task performance (Cabeza et al., 2002; 2018). There is also evidence of increased parietal activation in older adults in cognitively demanding tasks (e.g. DiGirolamo et al., 2001; Jimura \& Braver, 2010, Nielson, Langenecker, \& Garavan, 2002; Nielson et al., 2004; Vallesi, McIntosh, \& Stuss, 2011; Zhu, Zacks, \& Slade, 2010, Heuninckx, Wenderoth, Debaere, Peeters, \& Swinnen, 2005; Heuninckx, Wenderoth, \& Swinnen, 2008, Langenecker, Nielson, \& Rao, 2004; Prakash et al., 2009; Zysset, Schroeter, Neumann, \& Yves von Cramon, 2007). Huang, Polk, Goh \& Park (2011) showed that this 
1 increase in activation serves a compensatory function, as it was associated with improved

2 performance in resolving interference.

The current study therefore aimed to determine whether brain areas associated with

4 average reward are associated with reward frequency, and to assess whether there are age-based

5 differences in activation that reflect age-based differences in decision making strategies. We also

6 aimed to determine whether there is evidence of compensation in either prefrontal or parietal

7 regions in older adults. To achieve this aim, we used fMRI combined with model-based analyses

8 to examine the neural bases of decision-making in older and younger adults while performing the

9 SGT. We expected that older adults would engage in more reactive decision-making behavior

10 than younger adults. That is, older adults would be more likely to base their choices on the

11 outcome they received on the previous trial, whereas younger adults would be more likely to

12 base their choices on expected values integrated across a longer sequence of prior outcomes. In a

13 model-based fMRI analysis approach, reinforcement learning models are first fit to the

14 behavioral data. Model-derived components such as EVs and prediction errors are then used as

15 regressors in order to identify brain regions whose activity is associated with those components.

16 The benefit of this approach over traditional fMRI is that it provides a theoretically grounded

17 way of interpreting fMRI data, as it allows us to identify how cognitive processes are represented

18 in specific brain regions, rather than simply identifying where in the brain these processes occur

19 (O’Doherty, Hampton \& Kim, 2007; Glascher \& O’Doherty, 2010). Based on previous work, we

20 expected greater activation associated with expected values in the ventral striatum and PFC

21 regions involved in value based decision making, as well as greater PE-related activation in the

22 striatum (Hare et al., 2008; Rangel et al., 2008; Samanez-Larkin et al., 2014; Worthy et al., 23 2016). 


\section{Behavioral results}

Optimal choices (proportion of $\mathrm{C}$ and $\mathrm{D}$ deck choices) across four 25-trial blocks of the

4 SGT are shown in Figure 1. Performance was analyzed with a 2 (age group) x 4 (block) mixed measures ANOVA. There was a significant linear effect of block, $F(1,101)=14.52, p<.001, \eta^{2} p$

$6=.126$, indicating an increase in optimal choices as the task progressed. There was no significant

7 main effect of age group, $F(1,101)=.350, p=.555, \eta^{2} p=.003$. However, there was a significant

8 interaction between age group and the linear effect of block, $F(1,101)=9.19, p=.003, \eta^{2}=$

$9 \quad .083$. To further examine this interaction, we separately assessed the effect of block for each age

10 group. There was a significant linear effect of block for the younger adults, $F(1,49)=14.19, p<$.

$11001, \eta_{p}^{2}=.225$, but this effect did not reach significance for the older adults, $F(1,52)=0.724, p=$

$12.399, \eta^{2}{ }_{p}=.014$. This indicates that younger adults learned to select the optimal options more

13 frequently as the task progressed, whereas older adults did not show much improvement.

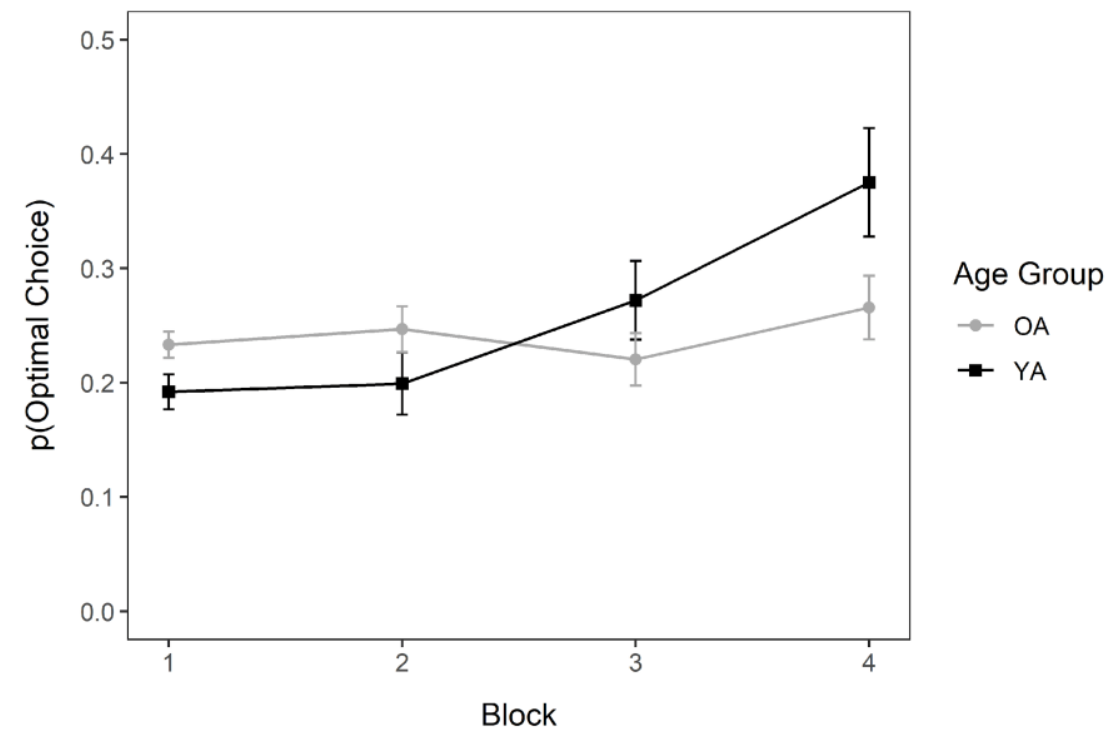

Figure 1. Mean optimal choices across four 25-trial blocks of the Soochow Gambling Task for older and younger adults. Younger adults (YA) show an improvement in optimal choices across the duration of the task, while older adults (OA) maintain suboptimal choices. 


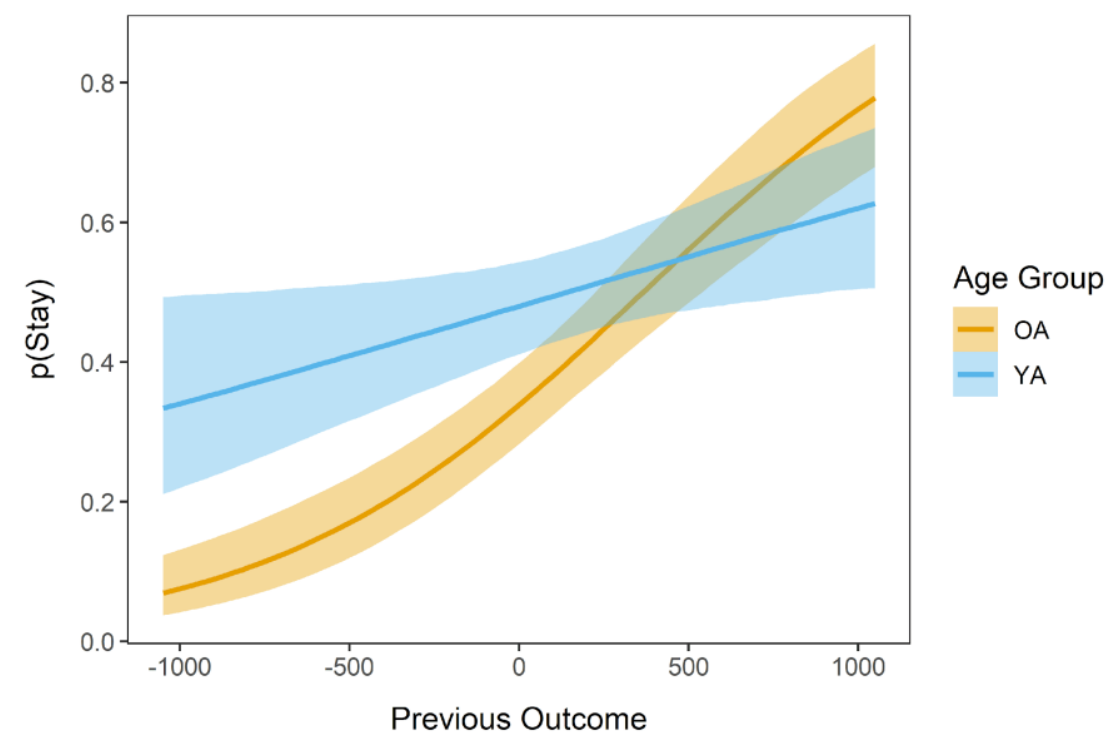

Figure 2. Probability of stay choices based on the outcome on the previous trial for younger adults (YA) and older adults (OA).

To examine whether older adults were more reactive decision-makers than younger

2 adults, we compared the probability of staying and switching based on the preceding outcome.

3 We hypothesized that, in general, participants would be more likely to stay if they received a

4 reward on the previous trial, and would be more likely to switch if they experienced a loss. If

5 older adults are more reactive decision-makers, they may be more likely to switch following

6 losses than younger adults.

To assess this hypothesis we estimated a mixed-effects model using the brms package in

$8 \mathrm{R}$ (Bürkner, 2017; 2018) to predict the probability of repeating the same choice (stay vs. switch),

9 based on the outcome of the previous trial, and age group. There was an overall tendency to

10 repeat choices that had higher reward on the previous trial $(B=0.002$, odds ratio $=1.002 ; 95 \%$

$11 \mathrm{CI}=[0.001,0.002])$. Younger adults were also more likely to repeat previous choices than older

12 adults $(B=0.67$, odds ratio $=1.956,95 \% \mathrm{CI}=[0.23,0.95])$. There was also an interaction

13 between previous outcome and age group, indicating a greater responsivity to the previous 
outcome in older adults than younger adults $(B=-0.001$, odds ratio $=0.999,95 \% \mathrm{CI}=-0.002$, to stay with the same option following larger gains.

\section{Modeling results}

We focused on the comparison of Delta and PE-Decay models in order to dissociate expected values that were computed based on average rewards and expected values based on the frequency of positive and negative outcomes. The Delta model represents a more optimal strategy, as it bases expected values on the average payoffs provided by each option. The PEDecay model represents a sub-optimal strategy, in which only the frequency of gains and losses is considered, and magnitude of gains and losses is ignored. The EVs produced by these models were not highly correlated within-subjects $(r=.003, p=.738$, see Figure 3$)$, and are therefore ideal as regressors for fMRI analyses. For consistency with previous research, we also fit two versions of the PVL model, using both a delta-reinforcement updating rule (PVL-delta) and a decay-reinforcement updating rule (PVL-decay). These models did not fit the data as well as the PE-Decay model, and the EVs produced by the PVL-Decay model were more highly correlated with the Delta model than the EVs produced by the PE-Decay model. ${ }^{1}$ The fits of these models are therefore reported in the Supplementary Material, but are not included as regressors in the

\footnotetext{
${ }^{1}$ The reason we did not compare the PVL-Decay model to the PVL-Delta model is that both of these models have a shape parameter that allows for exponential discounting of reward magnitudes. This means both models can account for choice behavior that is driven only by the frequency of reward provided by each option, and not the magnitude. The basic Delta model does not have a shape parameter, and the magnitude of past rewards is not discounted. This makes the basic Delta model a valuable comparator against models that assume that the magnitude of rewards is discounted, and choices are based primarily on the frequency of gains versus losses.
} 


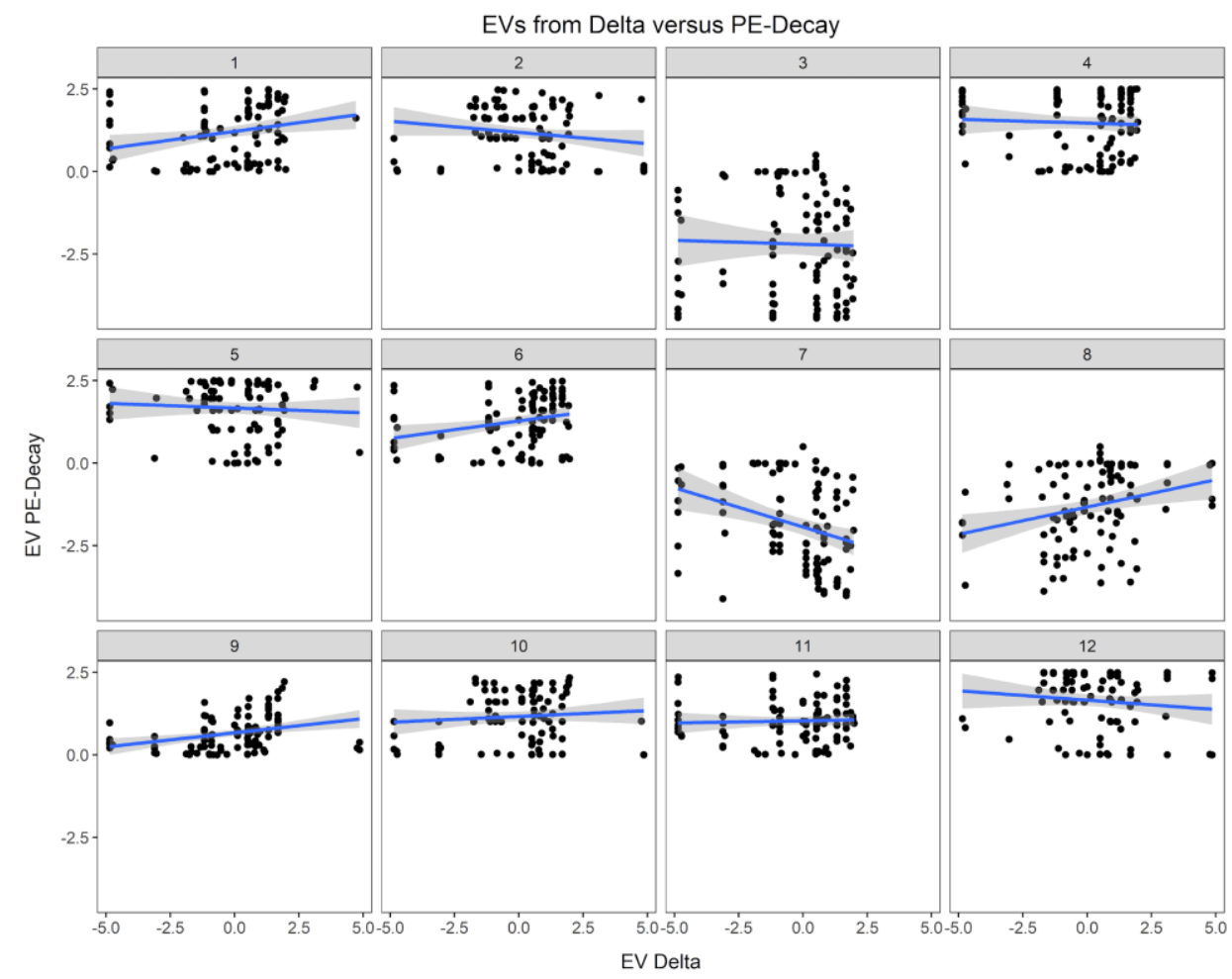

Figure 3. Correlations between expected values from Delta and PE-Decay models for 12 participants.

Table 2

BIC values and best-fitting parameter values for Delta and PE-Decay models. Standard deviations are shown in brackets.

\begin{tabular}{llll}
\hline & Younger adults & Older adults & Average \\
\hline $\begin{array}{l}\text { BIC values } \\
\text { Delta }\end{array}$ & $278.08(8.34)$ & $274.56(9.08)$ & $276.27(8.86)$ \\
PE-Decay & $234.81(31.37)$ & $237.49(31.98)$ & $236.19(31.56)$ \\
Parameter estimates & & & \\
Delta & & & \\
$\alpha$ & $.37(0.43)^{*}$ & $0.67(0.37)^{*}$ & $0.53(0.42)$ \\
$c$ & $.09(0.12)$ & $0.22(0.67)$ & $0.16(0.49)$ \\
PE-Decay & & & \\
$\alpha$ & $0.43(0.39)$ & $0.39(0.35)$ & $0.41(0.37)$ \\
$\lambda$ & $1.29(1.75)^{*}$ & $2.05(1.90)^{*}$ & $1.68(1.86)$ \\
$c$ & $0.50(0.33)$ & $0.48(0.33)$ & $0.49(0.32)$ \\
\hline
\end{tabular}

Note: *significant age difference at $\mathrm{p}<.05$. 
We fit each model to each individual participant's data by maximizing the log-likelihood

of the model's next step ahead predictions, and used Bayesian Information Criterion (BIC;

Schwarz, 1978) to compare model fits. Overall, the PE-Decay model provided a much better fit than the Delta model $(\triangle \mathrm{BIC}=40.08)$. From the behavioral data, it is clear that participants did not use the more optimal strategy represented by the Delta model. Figure 4 shows simulated optimal choices predicted by each model. The Delta model predicts a preference for the good decks that emerges quickly, while the PE-Decay model predicts persistent choice of the bad decks. The best-fitting parameters derived from each model are shown in Table 2. This table also presents the best fitting parameters for older and younger adults separately. For the Delta model, the best-fitting parameter for learning rate $(\alpha)$ was higher for older adults than younger adults, indicating a greater weight to recent outcomes in older adults. The same pattern was not evident in best-fitting decay rate parameters. However, older adults were more loss averse (mean $\lambda=$ 2.05) compared to younger adults (mean $\lambda=1.29), t(101)=2.10, p=.038, d=.413$.

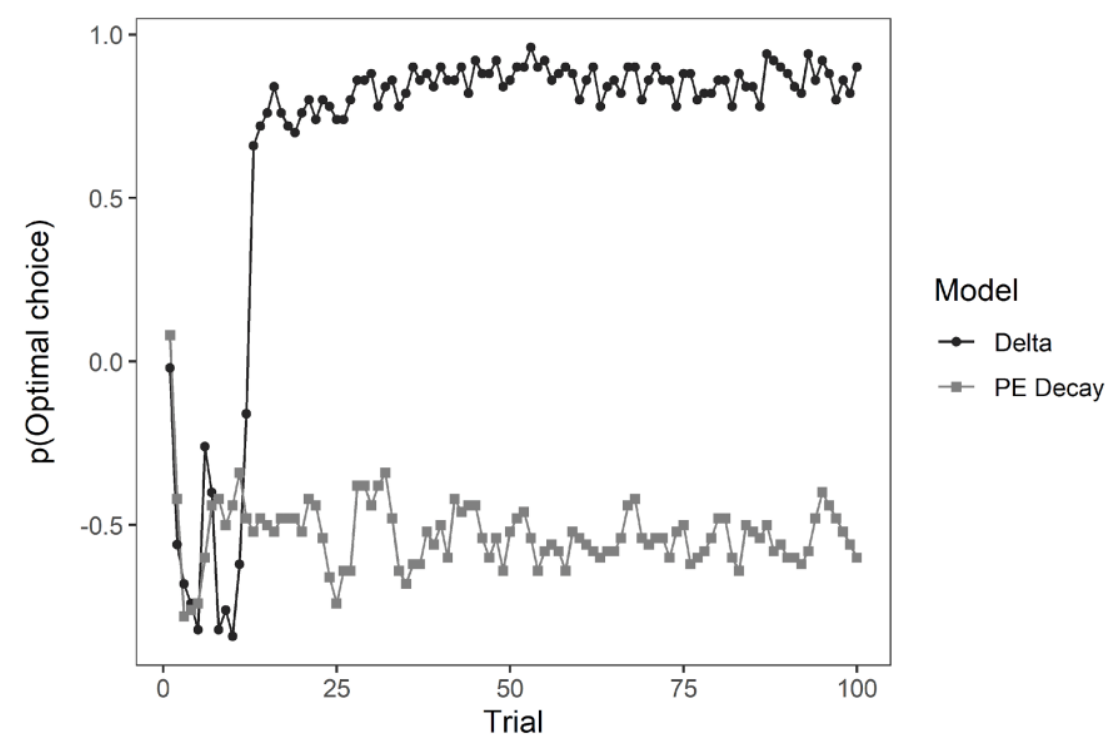

Figure 4. Simulated optimal choices across the SGT predicted by the Delta model and PE Decay model. The Delta model very quickly prefers the optimal decks after a large loss, while the PE Decay models predicts persistent choice of the suboptimal decks. 
Model-based fMRI analysis

For each of the models, we computed expected values for the chosen option, as well as

3 prediction errors (the difference between outcomes received and the expected value), using each

4 model's best-fitting parameters. Activation correlated with expected value and prediction errors

5 from each model are reported below.

\section{$6 \quad$ Expected value activation}

To assess activation uniquely associated with each model's expected values, we entered

8 both as regressors in the fMRI regression model, activation $\sim$ Delta EV + PE-Decay EV. Note

9 that the results are very similar when EVs for each model were entered into separate regression

10 models (see Supplementary Material).

Expected Value Delta Activation

a) YA

$\mathrm{R}$

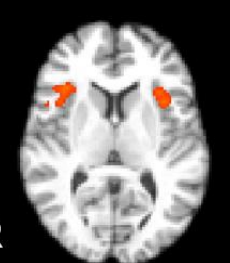

$z=8$

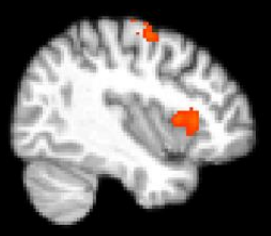

$x=40$

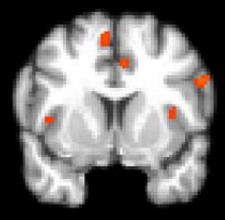

$y=10$

b) $Y A>O A$

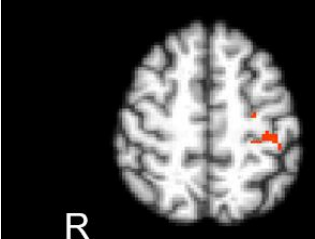

$\mathrm{R}$

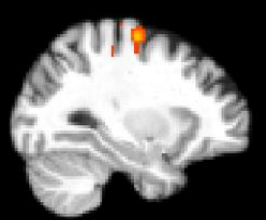

$$
x=-30
$$

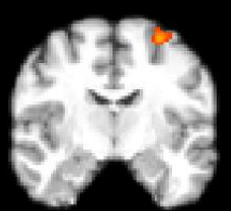

$y=-10$

Figure 5. a.) YA activation correlated with expected values from the delta model. b.) YA activation greater than OA activation correlated with expected values from the delta model. The delta model represents an optimal strategy for the task. 
Table 3

Delta model expected value unique activation.

\begin{tabular}{|c|c|c|c|c|c|c|c|c|}
\hline & \multirow[b]{2}{*}{ Region } & \multirow[b]{2}{*}{$\begin{array}{l}\text { Cluster } \\
\text { Index }\end{array}$} & \multirow[b]{2}{*}{ Voxels } & \multirow[b]{2}{*}{$\mathrm{P}$} & \multicolumn{4}{|c|}{ Maximum z-score $(\mathrm{mm})$} \\
\hline & & & & & Z-score & X & $\mathrm{Y}$ & $\mathrm{Z}$ \\
\hline \multirow[t]{6}{*}{ YA } & $\begin{array}{l}\text { L. precentral gyrus, post } \\
\text { central gyrus }\end{array}$ & 6 & 1269 & $<.001$ & 6.36 & -32 & -12 & 62 \\
\hline & $\begin{array}{l}\text { Supplementary motor } \\
\text { cortex }\end{array}$ & 5 & 526 & $<.001$ & 4.93 & -4 & -8 & 52 \\
\hline & $\begin{array}{l}\text { R. frontal operculum } \\
\text { cortex, insula, OFC }\end{array}$ & 4 & 355 & $<.001$ & 4.55 & 36 & 20 & 12 \\
\hline & $\begin{array}{l}\text { L. OFC, } \\
\text { insula, frontal operculum } \\
\text { cortex }\end{array}$ & 3 & 214 & $<.001$ & 4.31 & -32 & 28 & 2 \\
\hline & R. precentral gyrus & 2 & 197 & $<.001$ & 4.92 & 42 & -6 & 64 \\
\hline & L. precentral gyrus & 1 & 179 & $<.001$ & 4.59 & -54 & 4 & 30 \\
\hline \multirow[t]{2}{*}{$\mathrm{YA}>\mathrm{OA}$} & L. postcentral gyrus & 2 & 244 & $<.001$ & 4.75 & -36 & -38 & 40 \\
\hline & L. precentral gyrus & 1 & 116 & .005 & 5.61 & -32 & -12 & 64 \\
\hline
\end{tabular}

Note: $\mathrm{YA}=$ younger adults; $\mathrm{OA}=$ older adults; $\mathrm{R}=$ right; $\mathrm{L}=$ left; Frontal orbital cortex functionally labeled as OFC.

Delta model EV related activation. Table 3 lists regions of activation in younger adults, and regions where activation was significantly greater for younger adults than older adults. As the Delta model represents the more optimal strategy, these are the regions that are most active when selecting the optimal options. In younger adults (Figure 5a), Delta model expected values were associated with activation in regions involved in decision making, including the right and

6 left orbital frontal cortex (OFC), operculum cortex and the insula. There was also associated

7 activation in motor/pre-motor regions, including the precentral and post-central gyrus, and

8 supplementary motor cortex. Younger adults showed greater activation in the left precentral and 9 postcentral gyrus than older adults (Figure 5b).

11 correlated with expected values from the PE-Decay model for younger adults. These regions are

12 listed in Table 4. There was significant deactivation in frontoparietal regions as a function of 
1 expected value. That is, younger adults showed greater activation in these cognitive control

2 regions when expected value for the PE-Decay model was low. The PE-Decay model's expected

3 values will be lower for the good decks than the bad decks, as these decks provide more frequent

4 losses than gains. Thus, these regions were active in younger adults when they were selecting

5 from the good decks.

Table 4

Expected value PE Decay negative correlation.

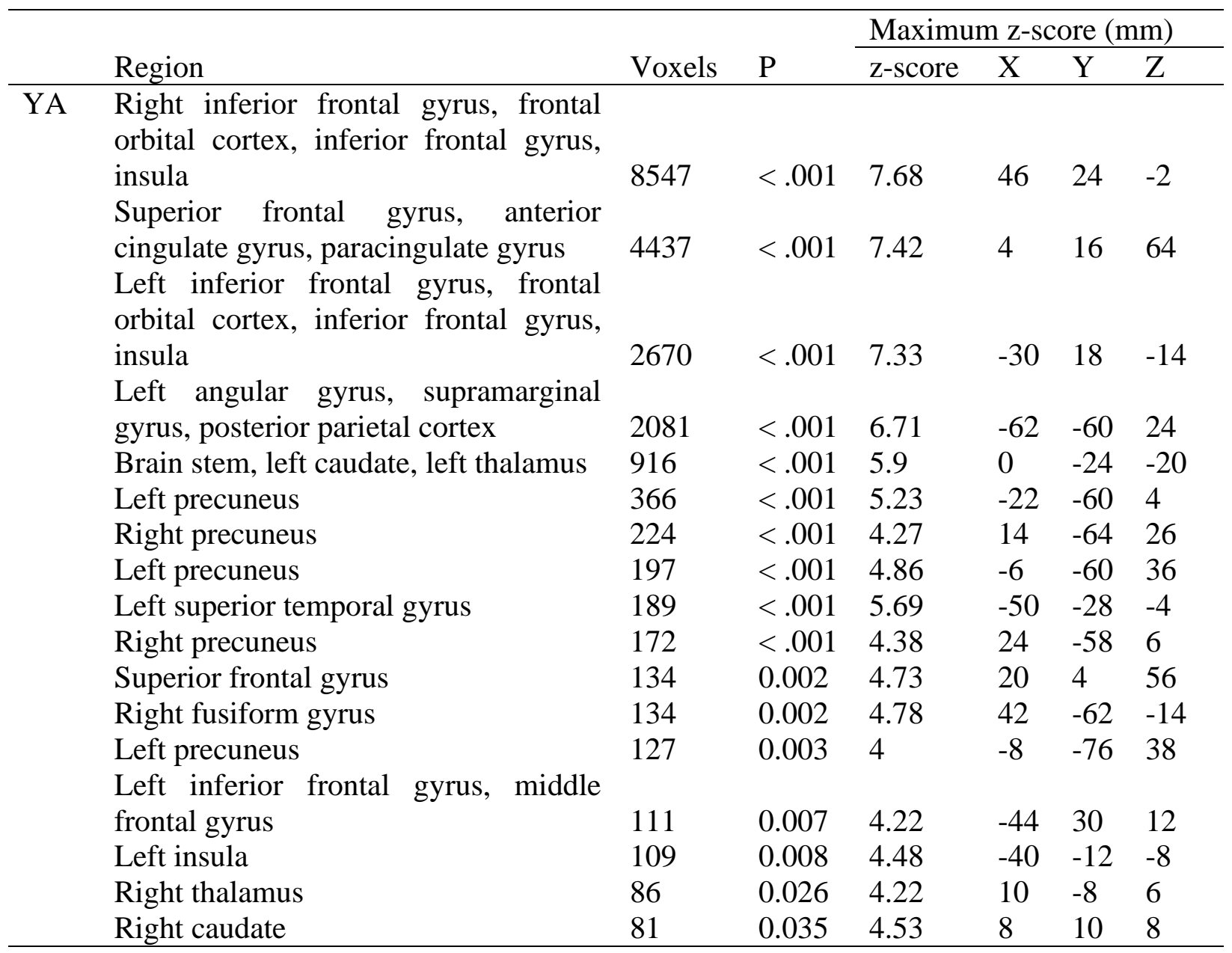

Note: Frontal orbital cortex functionally labeled as OFC, middle frontal gyrus functionally labeled as DLPFC. 


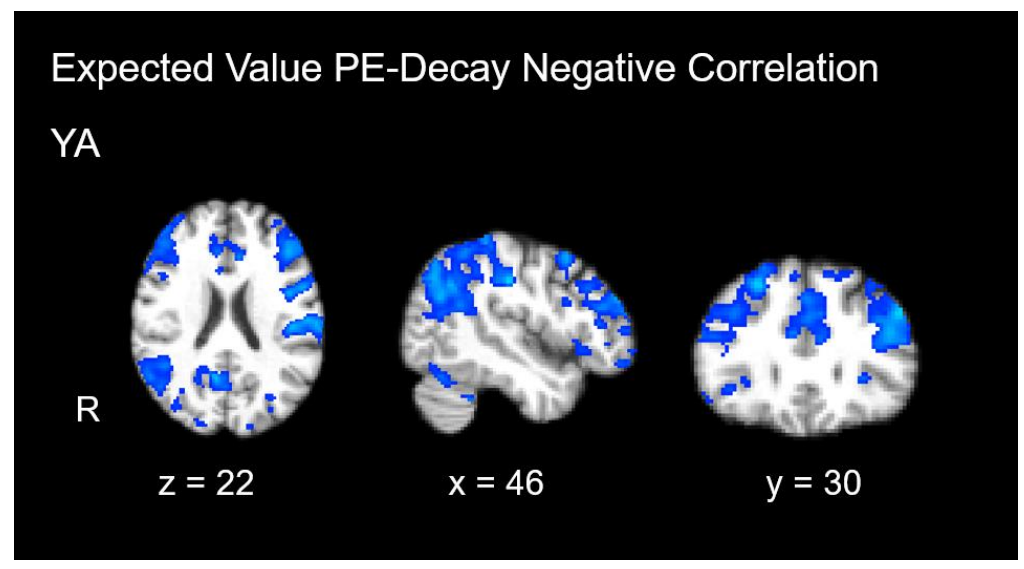

Figure 6. YA activation negatively correlated with expected values from the PE-Decay model. The PE-Decay model represents a sub-optimal strategy for the task. Thus, these regions were active when young adults made choices inconsistent with the sub-optimal, frequency-based strategy.

Table 5 lists regions where PE-Decay model EV related activation was greater for older

2 adults than younger adults, including greater bilateral activation in the DLPFC (see Figure 7).

3 Older adults also showed greater activation in the right and left frontal pole, left inferior frontal

4 gyrus, insula, and OFC than younger adults. This activation, combined with the negative

5 correlation with activation in younger adults, suggests that older adults may have been engaging

6 these regions to track which options had recently given gains versus losses.

\section{Expected Value PE-Decay Activation}

\section{$\mathrm{OA}>\mathrm{YA}$}

R

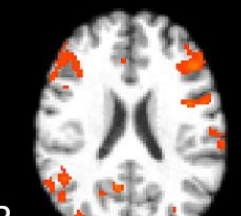

$z=22$

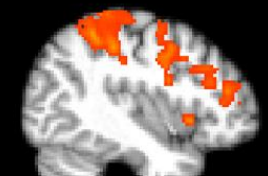

$x=-42$

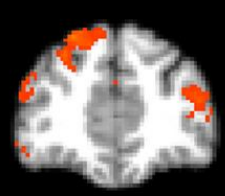

$y=44$

Figure 7. Greater activation for OA than for YA correlated with expected values from the PEDecay model. 
Table 5

PE-Decay model expected value unique activation.

\begin{tabular}{|c|c|c|c|c|c|c|c|c|}
\hline & \multirow[b]{2}{*}{ Region } & \multirow[b]{2}{*}{$\begin{array}{l}\text { Cluster } \\
\text { Index }\end{array}$} & \multirow[b]{2}{*}{ Voxels } & \multirow[b]{2}{*}{$\mathrm{P}$} & \multicolumn{4}{|c|}{ Maximum z-score (mm) } \\
\hline & & & & & Z-score & $\mathrm{X}$ & $\mathrm{Y}$ & $\mathrm{Z}$ \\
\hline \multirow[t]{20}{*}{$\mathrm{OA}>\mathrm{YA}$} & $\begin{array}{l}\text { Precuneus, lateral } \\
\text { occipital complex } \\
\text { (superior division), } \\
\text { posterior parietal cortex }\end{array}$ & 20 & 4872 & $<.001$ & 5.19 & 2 & -58 & 58 \\
\hline & $\begin{array}{l}\text { L. precentral gyrus, } \\
\text { DLPFC, post central } \\
\text { gyrus }\end{array}$ & 19 & 1896 & $<.001$ & 4.74 & -38 & -4 & 64 \\
\hline & R. frontal pole, DLPFC & 18 & 1430 & $<.001$ & 5.38 & 22 & 40 & 50 \\
\hline & $\begin{array}{l}\text { R. angular gyrus, lateral } \\
\text { occipital cortex } \\
\text { (superior division) }\end{array}$ & 17 & 851 & $<.001$ & 4.35 & 46 & -64 & 26 \\
\hline & $\begin{array}{l}\text { Lingual gyrus, occipital } \\
\text { pole }\end{array}$ & 16 & 565 & $<.001$ & 4.68 & 0 & -90 & -6 \\
\hline & $\begin{array}{l}\text { L. inferior frontal gyrus, } \\
\text { frontal operculum } \\
\text { cortex, OFC, insula }\end{array}$ & 15 & 313 & $<.001$ & 4.59 & -44 & 16 & -4 \\
\hline & R. frontal pole (rostral) & 14 & 309 & $<.001$ & 4.78 & 14 & 68 & 2 \\
\hline & R. superior frontal gyrus & 13 & 276 & $<.001$ & 4.43 & 22 & 0 & 54 \\
\hline & $\begin{array}{l}\text { R. middle temporal } \\
\text { gyrus }\end{array}$ & 12 & 229 & $<.001$ & 4.15 & 66 & -52 & 0 \\
\hline & $\begin{array}{l}\text { Superior frontal gyrus, } \\
\text { paracingulate gyrus }\end{array}$ & 11 & 160 & $<.001$ & 4.33 & -6 & 24 & 44 \\
\hline & R. supramarginal gyrus & 10 & 144 & .001 & 4.31 & 50 & -22 & 34 \\
\hline & Cerebellum & 9 & 140 & .001 & 4.3 & 26 & -52 & -28 \\
\hline & $\begin{array}{l}\text { Posterior cingulate } \\
\text { gyrus }\end{array}$ & 8 & 138 & .001 & 4.36 & 4 & -30 & 30 \\
\hline & Occipital pole & 7 & 135 & .002 & 4.12 & 16 & -94 & 24 \\
\hline & R. frontal pole (lateral) & 6 & 125 & .003 & 4.06 & 48 & 38 & -2 \\
\hline & L. fusiform gyrus & 5 & 116 & .004 & 4.02 & -24 & -74 & -14 \\
\hline & Anterior cingulate gyrus & 4 & 114 & .005 & 4.01 & 4 & 36 & 10 \\
\hline & $\begin{array}{l}\text { R. inferior temporal } \\
\text { gyrus }\end{array}$ & 3 & 98 & .011 & 4.4 & 56 & -22 & -26 \\
\hline & Lingual gyrus & 2 & 76 & .037 & 4.03 & 4 & -70 & -12 \\
\hline & $\begin{array}{l}\text { R. middle temporal } \\
\text { gyrus }\end{array}$ & 1 & 73 & .044 & 4.03 & 60 & -6 & -28 \\
\hline
\end{tabular}

Note: $\mathrm{YA}=$ younger adults; $\mathrm{OA}=$ older adults; $\mathrm{R}=$ right; $\mathrm{L}=$ left; Frontal orbital cortex functionally labeled as OFC, middle frontal gyrus functionally labeled as DLPFC. 


\section{Prediction error related activation}

Prediction errors produced by the Delta and PE-Decay models were substantially

2 overlapping, $r=.85, p<.001$. Figure 8 shows correlations between the models' prediction errors

3 for several participants. Table 6 lists regions of activation associated with Delta model prediction

4 errors and Table 7 lists regions of activation associated with PE-Decay model prediction errors.

5 In younger adults (Figure 9a, Figure 10a), for both Delta and PE-Decay prediction errors, there

6 was activation in the ventral striatum (caudate, nucleus accumbens and putamen), which are

7 regions typically found to be activated in response to prediction error (Rodriguez, Aron, \&

8 Poldrack, 2006). There was also activation in the precentral and postcentral gyrus. Table 8 shows

9 activation negatively correlated with prediction error from the Delta model (see Figure 11).

10 Younger adults showed a similar pattern of deactivation in frontoparietal areas with increasing

11 prediction error to that seen with PE-Decay expected value.

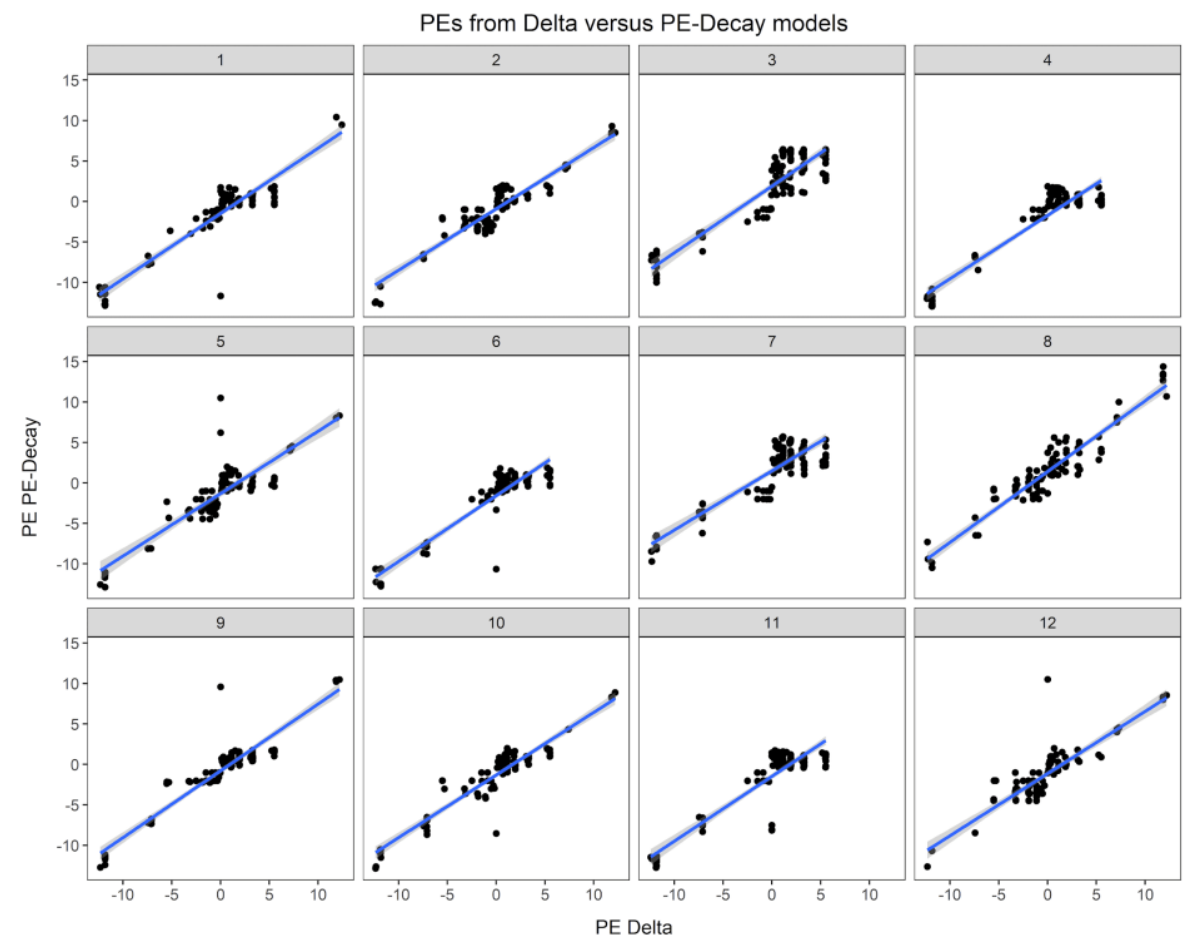

Figure 8. Correlations between Delta model prediction errors and PE-Decay model prediction errors for 12 random subjects. 


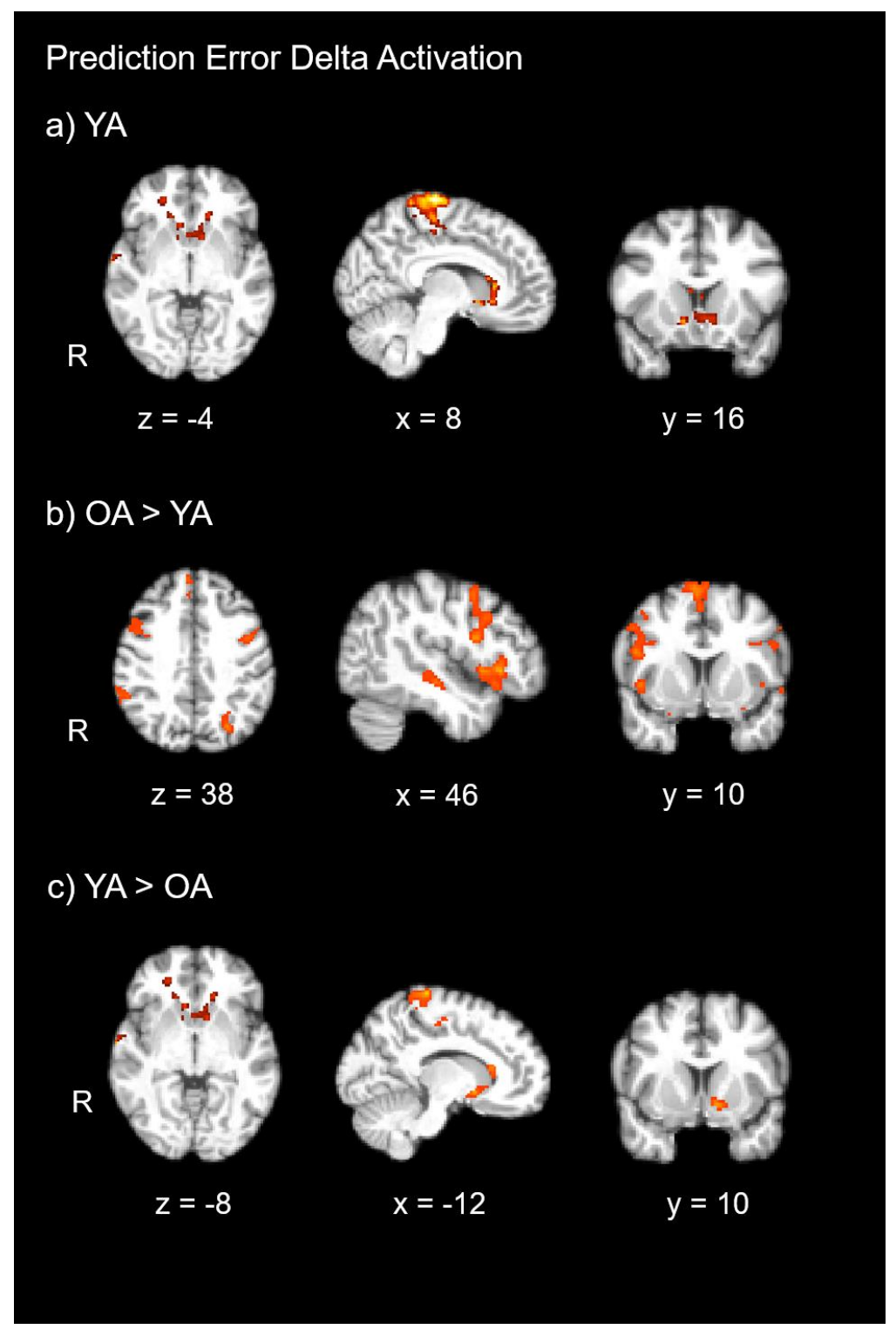

Figure 9. Prediction error related activation for the delta model. a.) younger adults. b.) older adults greater than younger adults. c.) younger adults greater than older adults. 
Table 6

Delta model prediction error activation.

\begin{tabular}{|c|c|c|c|c|c|c|c|c|}
\hline & \multirow[b]{2}{*}{ Region } & \multirow[b]{2}{*}{ Cluster Index } & \multirow[b]{2}{*}{ Voxels } & \multirow[b]{2}{*}{$\mathrm{P}$} & \multicolumn{4}{|c|}{ Maximum z-score (mm) } \\
\hline & & & & & Z-score & $\mathrm{X}$ & $\mathrm{Y}$ & $\mathrm{Z}$ \\
\hline \multirow[t]{10}{*}{ YA } & Precentral gyrus, postcentral gyrus & 10 & 2323 & $<.001$ & 7.15 & 2 & -26 & 64 \\
\hline & Nucleus accumbens, putamen, caudate & 9 & 991 & $<.001$ & 6.08 & 12 & 10 & -8 \\
\hline & R. precentral gyrus, superior parietal lobule & 8 & 612 & $<.001$ & 5.67 & 36 & -22 & 58 \\
\hline & Tail of caudate, right ventricle & 7 & 344 & $<.001$ & 4.75 & 16 & -36 & 22 \\
\hline & Tail of caudate, left ventricle & 6 & 229 & $<.001$ & 4.57 & -28 & -16 & 32 \\
\hline & Tail of caudate, left ventricle & 5 & 214 & $<.001$ & 5.1 & -22 & -36 & 16 \\
\hline & Superior temporal gyrus & 4 & 129 & 0.003 & 5.29 & 66 & -8 & -2 \\
\hline & Occipital pole & 3 & 112 & 0.007 & 4.34 & 20 & -90 & 20 \\
\hline & Occipital pole & 2 & 96 & 0.015 & 4.24 & -24 & -92 & 14 \\
\hline & Frontal pole & 1 & 80 & 0.037 & 4.72 & -8 & 70 & 10 \\
\hline \multirow[t]{11}{*}{$\mathrm{OA}>\mathrm{YA}$} & Superior frontal gyrus, paracingulate cortex & 11 & 1522 & $<.001$ & 5.46 & 4 & 14 & 64 \\
\hline & R. OFC, insula, frontal operculum, inferior frontal gyrus & 10 & 928 & $<.001$ & 5.25 & 52 & 18 & 0 \\
\hline & L. OFC, insula, frontal operculum, inferior frontal gyrus & 9 & 647 & $<.001$ & 5.34 & -30 & 20 & -14 \\
\hline & R. DLPFC, inferior frontal gyrus, precentral gyrus & 8 & 546 & $<.001$ & 5.08 & 42 & 6 & 48 \\
\hline & R. middle temporal gyrus, superior temporal gyrus & 7 & 354 & $<.001$ & 5.08 & 48 & -20 & -10 \\
\hline & R. angular gyrus, supramarginal gyrus & 6 & 264 & $<.001$ & 5.06 & 62 & -52 & 24 \\
\hline & L. DLPFC, inferior frontal gyrus, precentral gyrus & 5 & 224 & $<.001$ & 4.48 & -46 & 2 & 40 \\
\hline & L. lateral occipital cortex, superior division & 4 & 142 & 0.002 & 4.51 & -28 & -72 & 38 \\
\hline & L. angular gyrus, supramarginal gyrus & 3 & 117 & .005 & 4.94 & -60 & -58 & 26 \\
\hline & Brainstem & 2 & 113 & .006 & 4.99 & -2 & -28 & 0 \\
\hline & L. middle temporal gyrus, superior temporal gyrus & 1 & 76 & .046 & 4.44 & -50 & -26 & -4 \\
\hline \multirow[t]{5}{*}{$\mathrm{YA}>\mathrm{OA}$} & Precentral gyrus, postcentral gyrus & 5 & 1215 & $<.001$ & 5.94 & 2 & -26 & 62 \\
\hline & Precentral gyrus & 4 & 279 & $<.001$ & 4.72 & 38 & -22 & 58 \\
\hline & L. nucleus accumbens, putamen & 3 & 204 & $<.001$ & 4.82 & -12 & 10 & -8 \\
\hline & Caudate & 2 & 115 & .006 & 4.29 & 12 & 26 & 4 \\
\hline & Superior temporal gyrus & 1 & 88 & .024 & 5.1 & 66 & -8 & -2 \\
\hline
\end{tabular}

Note: $\mathrm{YA}=$ younger adults; $\mathrm{OA}=$ older adults; $\mathrm{R}=$ right; $\mathrm{L}=$ left; Frontal orbital cortex functionally labeled as OFC, middle frontal gyrus functionally labeled as DLPFC. 
Table 7

PE-Decay model prediction error activation.

\begin{tabular}{|c|c|c|c|c|c|c|c|c|}
\hline & \multirow[b]{2}{*}{ Region } & \multirow[b]{2}{*}{$\begin{array}{l}\text { Cluster } \\
\text { Index }\end{array}$} & \multirow[b]{2}{*}{ Voxels } & \multirow[b]{2}{*}{$\mathrm{P}$} & \multicolumn{4}{|c|}{ Maximum z-score (mm) } \\
\hline & & & & & Z-score & $\mathrm{X}$ & $\mathrm{Y}$ & $\mathrm{Z}$ \\
\hline \multirow[t]{10}{*}{ YA } & Precentral gyrus, post central gyrus & 10 & 4133 & $<.001$ & 6.98 & 0 & -26 & 66 \\
\hline & R. nucleus accumbens, caudate & 9 & 869 & $<.001$ & 5.94 & 12 & 10 & -8 \\
\hline & R. occipital pole & 8 & 237 & $<.001$ & 4.76 & 22 & -92 & 18 \\
\hline & L. cerebral white matter & 7 & 214 & $<.001$ & 4.66 & -28 & -14 & 32 \\
\hline & R. superior temporal gyrus & 6 & 179 & $<.001$ & 5.9 & 64 & -4 & -2 \\
\hline & L. superior temporal gyrus & 5 & 155 & .001 & 5.59 & -64 & -14 & 4 \\
\hline & R. cerebral white matter & 4 & 133 & .003 & 4.38 & 22 & -28 & 24 \\
\hline & R. precentral gyrus & 3 & 123 & .005 & 4.7 & 52 & 0 & 28 \\
\hline & L. cerebral white matter, putamen & 2 & 120 & .005 & 4.57 & -32 & -14 & 12 \\
\hline & R. cerebral white matter, caudate & 1 & 95 & .019 & 4.27 & 18 & -6 & 28 \\
\hline \multirow[t]{8}{*}{$\mathrm{OA}>\mathrm{YA}$} & Superior frontal gyrus & 8 & 717 & $<.001$ & 5.02 & 2 & 12 & 68 \\
\hline & R. inferior frontal gyrus, insula, OFC & 7 & 384 & $<.001$ & 4.37 & 44 & 12 & -2 \\
\hline & L. OFC, frontal operculum cortex & 6 & 282 & $<.001$ & 4.45 & -30 & 18 & -14 \\
\hline & R. middle temporal gyrus & 5 & 239 & $<.001$ & 4.84 & 54 & -20 & -10 \\
\hline & Superior frontal gyrus & 4 & 98 & .016 & 4.12 & 6 & 50 & 36 \\
\hline & Thalamus, brain stem & 3 & 95 & .019 & 4.2 & -2 & -28 & 0 \\
\hline & R. DLPFC & 2 & 93 & .021 & 4.06 & 38 & 2 & 46 \\
\hline & R. angular gyrus & 1 & 91 & .023 & 4.14 & 62 & -50 & 26 \\
\hline \multirow[t]{5}{*}{$\mathrm{YA}>\mathrm{OA}$} & Precentral gyrus, post central gyrus & 5 & 1431 & $<.001$ & 5.89 & 12 & -22 & 74 \\
\hline & Post central gyrus & 4 & 476 & $<.001$ & 4.82 & 38 & -22 & 58 \\
\hline & L. nucleus accumbens & 3 & 236 & $<.001$ & 4.83 & -12 & 10 & -10 \\
\hline & R. nucleus accumbens & 2 & 128 & .004 & 6.62 & 12 & 10 & -8 \\
\hline & Superior temporal gyrus & 1 & 100 & .015 & 5.31 & 64 & -6 & -2 \\
\hline
\end{tabular}

Note: $\mathrm{YA}=$ younger adults; $\mathrm{OA}=$ older adults; $\mathrm{R}$ = right; $\mathrm{L}=$ left; Frontal orbital cortex functionally labeled as OFC, middle frontal gyrus functionally labeled as DLPFC. 
Table 8

Prediction error PE Decay negative correlation.

\begin{tabular}{|c|c|c|c|c|c|c|c|}
\hline & \multirow[b]{2}{*}{ Region } & \multirow[b]{2}{*}{ Voxels } & \multirow[b]{2}{*}{$\mathrm{P}$} & \multicolumn{4}{|c|}{ Maximum z-score (mm) } \\
\hline & & & & Z-score & $\mathrm{X}$ & Y & $\mathrm{Z}$ \\
\hline \multirow[t]{13}{*}{ YA } & Right/Left post central gyrus, & & & & & & \\
\hline & Right/Left supramarginal gyrus, & & & & & & \\
\hline & Right/left posterior parietal lobe & 25818 & $<.001$ & 6.55 & -44 & -34 & 46 \\
\hline & Right frontal pole, middle frontal & & & & & & \\
\hline & gyrus, inferior frontal gyrus & 5748 & $<.001$ & 6.25 & 22 & 40 & 50 \\
\hline & $\begin{array}{l}\text { Anterior cingulate gyrus, } \\
\text { paracingulate gyrus }\end{array}$ & 1710 & $<.001$ & 5.58 & -8 & 22 & 44 \\
\hline & Left inferior frontal gyrus, insula & 769 & $<.001$ & 4.9 & -50 & 18 & -2 \\
\hline & Right middle temporal gyrus & 392 & $<.001$ & 4.42 & 60 & -56 & 0 \\
\hline & Right inferior frontal gyrus, insula & 347 & $<.001$ & 4.48 & 34 & 24 & -4 \\
\hline & Right putamen, caudate & 282 & $<.001$ & 4.22 & 24 & 12 & 4 \\
\hline & Left thalamus & 259 & $<.001$ & 4.78 & -12 & -18 & 10 \\
\hline & Right middle temporal gyrus & 209 & $<.001$ & 5.7 & 62 & -4 & -26 \\
\hline & Right thalamus & 94 & 0.0145 & 4.3 & 22 & -30 & 8 \\
\hline
\end{tabular}

Note: Frontal orbital cortex functionally labeled as OFC, middle frontal gyrus functionally labeled as DLPFC.

For Delta model prediction error, there was greater left nucleus accumbens activation in

2 younger adults than older adults. For PE-Decay prediction error, there was greater bilateral

3 activation of nucleus accumbens in younger adults than older adults (Figure 10c). In older adults,

4 there was greater activation in more lateral PFC and posterior parietal regions compared to

5 younger adults (Figure 9b), indicating that older adults may not be tracking prediction error as

6 well as younger adults in the ventral striatum. This activation in lateral posterior parietal cortex

7 also correlated with optimal responding in the task within older adults (Figure 12). Regions of

8 activation that were greater for older adults than younger adults were similar to those associated

9 with PE-Decay model expected values, including right DLPFC, bilateral OFC and insula. This is

10 consistent with the idea that older adults are using short-term, working memory strategies to

11 remember which options recently led to positive versus negative prediction errors, while 
1 activation in the ventral striatum in younger adults indicate that they are using prediction errors

2 to update long-term expected values.

\section{Prediction Error PE-Decay Activation}

a) YA

$\mathrm{R}$
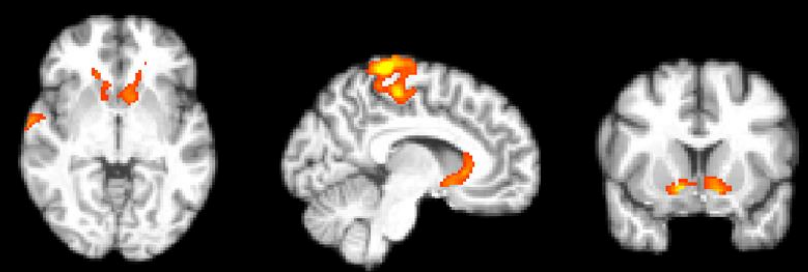

$z=-4$

$x=-8$

$y=-12$

b) $\mathrm{OA}>\mathrm{YA}$

$\mathrm{R}$
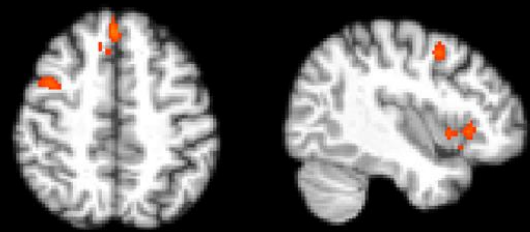

$z=48$

$x=42$

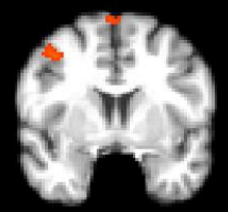

$$
y=4
$$

c) YA $>$ OA

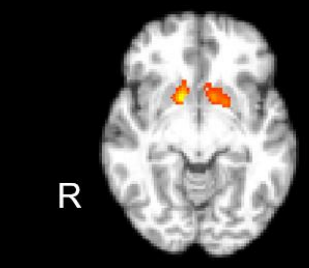

$$
z=-8
$$

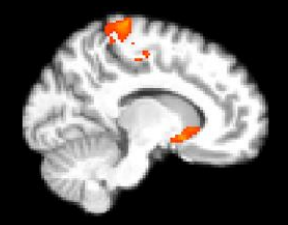

$$
x=-12
$$

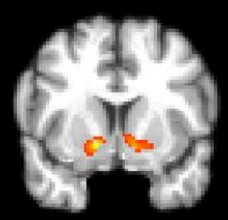

$$
y=10
$$

Figure 10. Prediction error related activation for the PE-Decay model. a.) younger adults. b.) older adults greater than younger adults. c.) younger adults less than older adults. 


\section{Prediction Error Delta Negative Correlation}

\section{YA}

$\mathrm{R}$
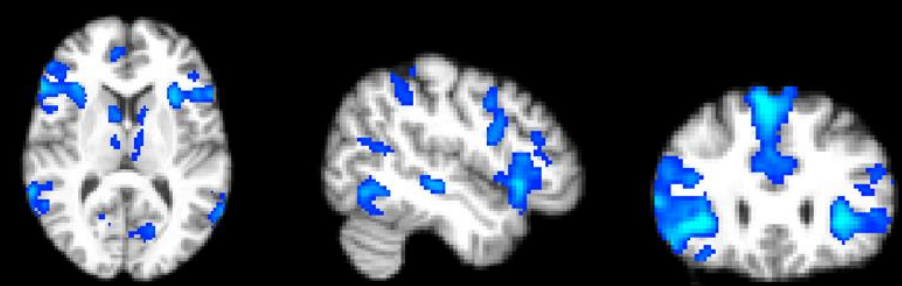

$$
z=10
$$

$$
x=-48
$$

$$
y=28
$$

Figure 11. Negative correlation with prediction errors from Delta model for younger adults.

\section{Prediction error correlated with optimal responding}

\section{OA}

$\mathrm{R}$
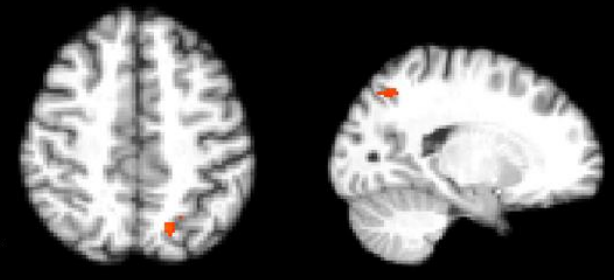

$$
z=46
$$

$$
x=-20
$$

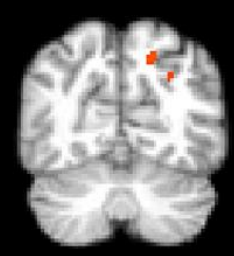

$$
y=-66
$$

Figure 12. Prediction error related activation from the Delta model correlated with optimal responding

\section{Discussion}

We compared performance of older and younger adults in a decision-making task that

3 dissociates average reward from frequency of gains versus losses. Overall, participants

4 performed poorly in this task, preferring the decks that provided small frequent gains but large

5 infrequent losses, which resulted in a negative net payoff. However, younger adults showed an

6 improvement in optimal choices across the duration of the task, while older adults maintained

7 their preference for the sub-optimal decks. This pattern of results suggests that younger adults 
1 were better able to adjust their choices based on average reward with experience. Older adults

2 appeared to be more reactive to recent outcomes than younger adults. Younger adults engaged

3 cognitive control networks to suppress negative prediction errors that resulted from picking the

4 good decks, which gave frequent losses. This may be why younger adults were less reactive to

5 recent events compared to younger adults, and why they showed better learning across the task.

$6 \quad$ The data from both younger and older adults in this task were better fit by the PE-Decay

7 model than the Delta model. The PE-Decay model tracks the number of positive versus negative

8 prediction errors, and will therefore value options with frequent gains more highly than frequent

9 losses, such that it predicts suboptimal performance in the task. In contrast, the Delta model

10 predicts more optimal performance based on average reward magnitude. The modeling results

11 suggest that decisions were based more on the frequency of positive versus negative outcomes

12 provided by each option, rather than the magnitude of outcomes, which resulted in poor

13 performance in the task. This is consistent with previous research that shows the SGT is better fit

14 by models that use decay updating rules (Ahn et al., 2008; Dai et al., 2015).

Consistent with previous research, older adults also appeared to be more reactive to

16 recent outcomes. In particular, older adults were more likely to switch choices following losses

17 than younger adults. This is in keeping with previous findings that older adults show greater

18 avoidance of negative outcomes and greater focus on negative feedback (Eppinger, Hammerer,

19 \& Li, 2011; Eppinger \& Kray, 2011; Frank \& Kong, 2008; Hämmerer, Li, Muller, \&

20 Lindenburger, 2011; Simon, Howard, \& Howard, 2010). The best-fitting loss aversion

21 parameters in the PE-Decay model were also higher for older adults than younger adults. Our

22 results therefore indicate a difference in behavioral strategy between older and younger adults in 
1 the SGT, similar to those found in some studies of the IGT (Wood et al., 2005). However, unlike

2 Wood et al., we saw both a difference in strategy and in performance.

Model-based fMRI analyses also indicate differences in activation associated with

4 expected values and prediction errors for older and younger adults. In younger adults, delta

5 model expected values, which predict more optimal responding, were associated with areas

6 implicated in decision making, including bilateral OFC, operculum cortex and insula. OFC has

7 been implicated in suppression or responses to previous outcomes, and this is consistent with

8 younger adults' reduced sensitivity to previous outcomes compared to older adults (Elliott et al.,

9 2000). Several regions showed greater activation associated with PE decay expected values for

older adults than younger adults. The PE-Decay model represents a sub-optimal strategy, based

11 on the frequency of gains versus losses, which fits closely with the pattern of behavior shown by

12 older adults in the task. Consistent with this result, older adults showed greater activation in

13 DLPFC associated with PE-Decay expected values, in accord with the hypothesis that DLPFC is

14 involved in accumulating information about past positive outcomes associated with each option.

15 This finding is consistent with prior studies in decision making (Worthy et al., 2016) and

16 working memory (Cappell et al., 2010; Hillary et al., 2006; Park \& Reuter-Lorenz, 2009). This

17 pattern of activation is also consistent with the idea that older adults are using short-term

18 memory strategies in which they remember which options led to gains and losses on recent trials,

19 and use this to guide their choices. However, younger adults showed a negative association

20 between frontoparietal activity and both prediction error and PE-Decay model expected values.

21 That is, there was greater activation in these regions when expected value and prediction error

22 was low. The good decks will have lower PE-Decay model expected values and prediction

23 errors, as they provide more frequent losses. Thus, the increased activation in these regions for 
1 older adults compared to younger adults may actually be due to younger adults tracking expected value and prediction error to a greater extent than older adults, with younger adults using more controlled processes on low expected value and prediction error trials to select the good decks even though they frequently provide losses resulting in negative prediction errors. Suppressing these negative prediction errors may have led to younger adults' better learning across the task.

The results for prediction error related activation in younger adults are particularly noteworthy. Previous studies have found activation in the ventral striatum related to Delta model prediction errors (Blair et al., 2006; Daw et al., 2006; Elliot e al., 2000; Hare et al, 2008; Pagnoni et al., 2002; Pessiglione et al., 2006). This has been taken as tacit neurobiological support for the Delta model's assumptions and predictions about behavior. Here, we also found activation in the ventral striatum associated with Delta model prediction errors. However, activation in this area was also associated with prediction errors from the PE-Decay model, and prediction errors produced by the PE-Decay model were highly correlated with those produced by the Delta model. Although the prediction errors were highly correlated, the expected values produced by the models were not strongly associated, and each model made distinct predictions about behavior in the SGT. We found that human behavior was more closely aligned with the predictions made by the PE-Decay model than those of the Delta model. Thus, the striatal activation related to Delta model prediction errors in previous studies does not necessarily support the idea that the Delta model is an accurate model of human decision-making. Instead, such activation may be indicative of prediction error related activation produced by applying a decay model. Support for computational models should therefore come from multiple sources, such as behavior, fMRI, and physiological responses as two models may be highly correlated on one metric such as prediction errors, but uncorrelated on another metric like expected value. 
Prediction error activity also differed between older and younger adults. We found

2

3

4 evidence of broader recruitment of prefrontal and parietal regions in older adults than younger adults. Activation in parietal regions were associated with optimal responding in the task, providing further evidence that activation in this area serves a compensatory function in older adults (Huang et al., 2012). Cabeza and colleagues (2018) have argued that increased activation in older versus younger adults must correlate with better performance on the task for the activation to be considered age-related neural compensation. Based on that criterion the enhanced prediction error related activity in parietal regions in older adults may be considered compensatory because it was correlated with performance. This enhanced prediction-error related activation may have led some older adults to use prediction errors more effectively to learn the long-term average rewards associated with the different choice options.

While Cabeza et al.'s (2018) criterion of improved performance may be good in some cases good performance is clearly defined, it's important to note that defining what is optimal in a given task is not always easy, particularly in decision-making tasks (Einhorn \& Hogarth, 1982). For example, one could design a task where attending to frequent rewards is the optimal strategy. In that case older adults' tendency to focus on frequency of reward more than younger adults might lead to broader activation of frontal parietal regions than younger adults as well as improved performance. We might interpret the increased activation in older adults related to implementing a frequency-based strategy as compensatory in that task, while in the current task we would not since attending to frequency is sub-optimal. Thus, older adults might be engaging in the same activation related to their strategy use, but whether it leads to improved performance is dependent on the optimal strategy in the task. 
Stern and colleagues made a similar point in their reply to Cabeza et al.’s 2018 paper. They view compensation as one of the many ways cognitive reserve can be implemented, and note that the success of compensation can be modulated by lifestyle variables. As we note above,

4 the success of compensation may be related to task, or other environmental variables as well.

5 Older adults may recruit a broad number of brain regions when implementing a sub-optimal

6 cognitive strategy. Whether this strategy is successful seems irrelevant to whether the enhanced

7 neural activation is viewed as evidence for neural compensation. Model-based fMRI, similar to

8 what we have conducted in this paper, may help identify the types of strategies that younger and

9 older adults are using, and exactly what brain regions younger and older adults are recruiting to

10 implement those strategies.

11 The SGT was designed to tease apart the confounds of average reward and gain-loss

12 frequency in the IGT (Bechara et al., 1994; 1997). In the IGT, healthy adults initially tend to first

13 prefer the high-gain-high-loss decks, but soon learn to choose the more advantageous low-gain-

14 low-loss decks. In this task, both good and bad decks provide a similar number of gains, but the

15 good decks provide fewer losses. Thus, it is unclear whether improved performance across the

16 IGT in healthy adults is guided by EV or gain-loss frequency (Lin, 2007; Chiu et al., 2008). The

17 finding that healthy older adults are more sensitive to recent outcomes and continue to prefer the

18 bad decks in the SGT suggests that gain-loss frequency is driving their decision-making. This is

19 important because it makes age-related differences in performance on the IGT difficult to

20 interpret. Some studies have found impaired IGT performance in older adults relative to younger

21 adults (Beitz, Salthouse \& Davis, 2014; Denburg, Tranel \& Bechara, 2005; Fein, McGillivray, \&

22 Finn, 2007; Isella et al., 2008; Rogalsky et al., 2012), while others have shown equivalent

23 performance, but using different strategies (Lamar \& Resnick, 2004; MacPherson, Phillips, \& 
1 Della Sala, 2002; Wood, Busemeyer, Koling, Cox, \& Davis, 2005). In particular, older adults

2 show greater recency biases, with greater forgetting of past outcomes (Wood et al., 2005). Older

3 adults also appear to display a loss frequency bias, showing a preference for options with a lower

4 frequency of punishment (Beitz et al., 2014; MacPherson et al, 2002).

Along with the previous studies, the results we report in the present study suggest that

6 older adults are consistently more reactive to recent outcomes when making decisions. Whether

7 this can be characterized as an age difference in strategy use with older adults employing a win-

8 stay-lose-shift type of strategy, changes in working memory capacity, or differences in in

9 selective attention to different features of the task such as gain-loss frequency are questions that

10 could be more directly examine in future studies. Many previous studies have focused only on

11 one or a few metrics of performance such as optimal choices, but there has been extensive

12 progress made in recent years on methods for RL and mixed effects modeling, and these types of

13 analyses can answer additional questions or corroborate conclusions based on other measures.

14 Our behavioral, modeling and fMRI results suggest that older adults may have a tendency to

15 focus mainly on the most recent outcomes, particularly recent losses. This tendency could be a

16 common cause for suboptimal decision-making behavior as people age. 


\section{Materials and methods}

\section{Participants}

Healthy younger and older adults were recruited from the Austin, Texas area. The study was advertised through posters, online forums, and recruitment events at aging conferences and senior recreation centers. Candidate participants were invited to participate in the study if they met the following inclusion criteria: 1) endorsed fewer than 8 items on the Pittsburgh Sleep Quality Inventory (PSQI), 2) endorsed fewer than 16 items on the Center for Epidemiological Studies Depression Scale (CESD) or fewer than 15 items on the Geriatric Depression Scale (GDS), 3) did not meet criteria for significant sleep disturbance or disorder, cardiovascular disease, and/or neurological or psychiatric disorders, and 4) were not currently taking sleep medication or psychoactive substances. Candidate participants were administered a neuropsychological battery assessing executive function and memory, and those who scored greater than two standard deviations from the age-adjusted norm were excluded from the study.

The data from fifty-three older adults (mean age $=67.7, S D=5.58$, range: $60-81,38$ female) and 50 younger adults (mean age $=21.3, S D=3.5$, range: $18-30,28$ female) were analyzed. Three additional participants were recruited but were excluded from the analyses for not meeting the neuropsychological assessment criteria (1), or incomplete SGT data sets (2). Participants were compensated for their participation in the study. Ethical approval was received from The University of Texas at Austin Institutional Review Board and prior written consent was obtained from all participants.

\section{Neuropsychological assessments}

All candidate participants were administered an abbreviated neuropsychological battery, including the following assessments: the Wechsler Adult Intelligence Scale IV (WAIS-IV) 
1

Vocabulary and Digit Span subtest, Trail Making Test A and B, and Psychomotor Vigilance

Test. Older adults were additionally administered the California Verbal Learning Test-II (CVLT-
II) and Controlled Oral Word Association Test (COWAT-FAS).

\section{Procedure}

Enrolled participants were administered a neuropsychological battery comprising assessments designed to test executive function and memory and a psychomotor vigilance task to measure arousal and attention. Eligible participants were fitted with an actigraph watch, which was worn on the left wrist for a minimum of 10 days. Participants also completed daily sleep surveys to document bed time, wake time, sleep quality, and any instances in which they removed the actigraph. All behavioral data were collected and managed using REDCap electronic data capture tools hosted at The University of Texas at Austin. Following the actigraphy period, participants underwent MRI scanning, during which T1w structural images and functional images were collected.

\section{MRI acquisition}

Imaging data was collected using a Siemens Skyra 3T scanner (TIM Systems, Siemens Medical Solutions, Erlangen, Germany) with a 32-channel head coil at the Biomedical Imaging Center at The University of Texas at Austin. Anatomical MRI volumes were acquired for coregistration with functional data using a 3D Multi-echo MPRAGE T1-weighted (T1w) sequence with the following parameters: $\mathrm{TR}=2530.0 \mathrm{~ms}, \mathrm{TE}=1.69,3.55,5.41$, and $7.27 \mathrm{~ms}, \mathrm{~T} 1=1100 \mathrm{~ms}$, $\mathrm{FOV}=256 \mathrm{~mm}^{2}, 176$ coronal slices, voxel size $1.0 \mathrm{~mm}^{3}$.

Participants viewed the stimuli via a back project screen and a mirror mounted on top of the head coil and responded with two four-button MR-compatible optical transmission devices, one held in each hand. Functional gradient echo EPI images were collected during two runs of the SGT 
1 task $(\mathrm{TR}=1500 \mathrm{~ms}, \mathrm{TE}=30 \mathrm{~ms}, 65$ axial slices oriented for best whole head coverage,

2 acquisition voxel size $=2 \times 2 \times 2 \mathrm{~mm}^{3}$, FOV 220x200 mm).

\section{Behavioral Task}

4 Participants completed two 50-trial scanning runs of the SGT. The reward structure for

5 each option is shown in Table 1. Participants were instructed that they would repeatedly select

6 from one of four decks of cards, and that they could gain or lose points on each draw. They were

7 given 2000 points to begin, and their goal was to try to finish with at least 2500 points.

8 Participants were told to do their best to maximize their gains and minimize their losses. On each

9 trial, four colored rectangles representing decks of cards were presented horizontally aligned on

10 the screen (See Figure 13), accompanied with the prompt "PICK A CARD". The point goal and

11 the point total were presented on the right side of the screen. Participants selected a deck by

12 pressing a corresponding number key. If participants responded within $2 \mathrm{~s}$, this was followed by

13 a variable fixation, feedback presented for $2 \mathrm{~s}$, and a variable inter-trial interval (ITI). During

14 feedback, the selected deck was presented in white, with the number of points gained or lost

15 displayed on the card. Gains were presented in black text, and losses presented in red text. If

16 participants responded slower than $2 \mathrm{~s}$, the fixation was replaced with the instruction "You must

17 respond sooner" and the feedback screen displayed only the point goal and total. 


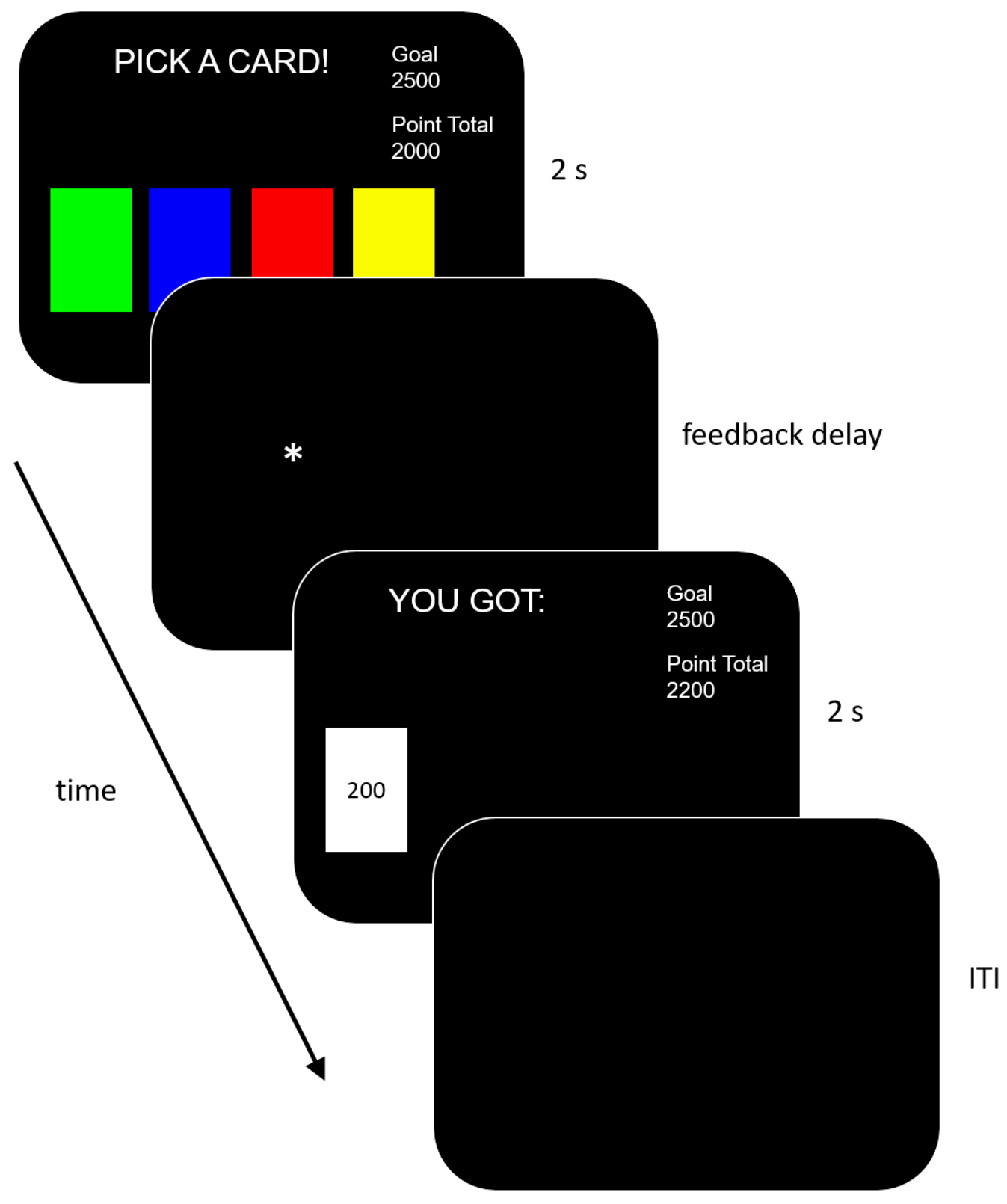

Figure 13. Schematic of the trial structure of the Soochow Gambling Task. Participants selected a card by pressing a corresponding key. This was followed by a variable delay before presenting feedback. The selected card was presented in white, and the number of points gained or lost were displayed on the card. The point goal and point total were displayed throughout the entirety of the task on choice and feedback screens. There was a variable inter-trial interval (ITI). If participants did not select a card within $2000 \mathrm{~ms}$, the fixation screen was replaced with "You must respond sooner", and no card was shown on the feedback screen. 


\section{Models}

We fit participants' behavioral data with two reinforcement learning models, a Delta model, and a Prediction-Error Decay model (PE-Decay), in order to dissociate expected values that were computed based on average reward and expected values based on the frequency of positive versus negative outcomes. The Delta model (Rescorla \& Wagner, 1972; Widrow \& Hoff, 1960; Williams, 1992) updates expected values based on prediction error: the difference between what was expected and what was received in a given instance. Delta model expected values will therefore approximate the average reward associated with each option. Expected values $(\mathrm{EV})$ in the delta rule model are calculated as:

$$
E V_{j}(t+1)=E V_{j}(t)+\alpha \cdot\left(r(t)-E V_{j}(t)\right) \cdot I_{j}
$$

where $I_{j}$ is an indicator value that is set to 1 if option $\mathrm{j}$ is selected on trial $\mathrm{t}$, and 0 otherwise.

Rewards $(r)$ are the points earned on trial t. Prediction error is represented by the portion of Equation 1 in parentheses, and is modulated by a learning rate parameter $(0 \leq \alpha \leq 1)$. Higher values of $\alpha$ indicate greater weight to recent outcomes, while lower values indicate less weight to recent outcomes. When $\alpha=0$ no learning takes place and expected values remain at their starting points, and when $\alpha=1$ expected values are equal to the last outcome received for each option. The predicted probability that option $j$ is chosen on trial $t$ is calculated using a Softmax rule:

$$
p\left|C_{j}(t)\right|=\frac{e^{\beta \cdot E V_{j}(t)}}{\sum_{1}^{N(j)} e^{\beta \cdot E V_{j}(t)}}
$$

where $\beta=3^{c}-1$, and $(0 \leq c \leq 5)$ is a log inverse temperature parameter that determines how consistently the option with the higher expected value is selected (Yechiam \& Ert, 2007). Lower values of $c$ provide more random choices, and as $c$ increases the option with the highest expected value is selected most often. Defining $\beta$ in this way allows it to take on a very large range of 
1 values (0-242), and is equivalent to setting a prior on beta with a truncated exponential

2 distribution.

The PE-Decay model used here is similar to that used in Pang et al (2017). In the PE-

4 Decay model, a prediction error (PE) is first computed as the difference between the reward 5 given on trial $\mathrm{t}, r(t)$, and the expected value for the chosen option, $i$ :

$$
P E=r(t)-E V_{i}(t)
$$

7 The prediction error is then used to update expected values for each $\mathrm{j}$ option on trial $\mathrm{t}+1$ :

$$
E V_{j}(t+1)=E V_{j}(t)+\alpha \cdot P E \cdot I_{j}
$$

9 Here, $(0 \leq \alpha \leq 1)$ is a learning rate parameter, where higher values indicate greater weight to 10 more recent events. I is a dummy variable coded as 1 if the option was chosen, or 0 otherwise, 11 such that only expected values for the chosen option is updated. Frequency value (FV) is then 12 updated and incremented based on expected value and prediction error:

$$
F V(t+1)=\left\{\begin{array}{l}
F V(t) \cdot(1-\alpha)+1, \text { if } P E>0 \\
F V(t) \cdot(1-\alpha)-\lambda, \text { if } P E<0
\end{array}\right.
$$

14 Such that FV would increment by 1 if prediction errors were positive, and would decrease by a 15 loss aversion parameter $(0 \leq \lambda \leq 5)$ if prediction errors were negative. The inclusion of this 16 parameter allows losses to have either more or less an effect than gains. The PE-Decay model 17 therefore simply tracks the cumulative instances of positive and negative prediction errors, and 18 does not consider the magnitude of rewards or losses. That is, a loss of 100 points will be treated 19 in the same way as a loss of 1,000 points. Frequency value is modulated by a decay rate 20 parameter, $(1-\alpha)$, where higher values of $\alpha$ indicate a higher rate of decay, and therefore 
1 greater weight to more recent events. Choice probabilities are calculated by entering FVs into

2 Equation 2 in place of EVs.

\section{$3 \quad$ fMRI Processing and Analysis.}

4 Pre-processed data (Esteban et al., 2018) were analyzed using a standard three-level general

5 linear model (GLM) analysis implemented in FSL's FEAT. The first-level models tested the

6 effect of task-related variables within single functional runs. All task related models included

7 constant EVs for the effect of choice and feedback parts of a trial and nuisance regressors for the

86 realignment parameters and their temporal derivatives. Model-based measures were included in

9 first-level models as parametric modulators. Five separate first-level models were run: one with

10 expected value and frequency value simultaneously modeled as modulators of choice, one model

11 where each of expected value and frequency value were modeled separately as modulators of

12 choice, and two models where PE from each model were modeled separately as modulators of

13 feedback. Second level models averaged effects of task variables across individual runs within

14 each participant using a fixed effects model. Third-level models tested whether task variables

15 were significant across participants using a mixed effects model for population inference. Final

16 statistical maps were corrected for multiple comparisons at $\mathrm{p}<.05$ using a Gaussian Random

17 Field Theory-based correction with a primary (cluster-forming) threshold of $\mathrm{z}=3.1(\mathrm{p}=.001$, 18 one-tailed). 


\section{Acknowledgements}

2 This work was supported by NIA grant AG043425 to DMS and DAW. The authors declare no 3 conflicts of interest.

5 H.J. Don helped design the study, analyzed the data, and wrote the paper; T. Davis analyzed the

6 data and edited the paper; K.L. Ray collected and analyzed the data, and edited the paper; M.C.

7 McMahon collected and analyzed the data, and edited the paper; A.C. Cornwall helped design

8 the study, programmed the study, and edited the paper; D.M. Schnyer helped designed the study,

9 oversaw data collection, and edited the paper. D.A. Worthy helped design the study, analyzed the 10 data, and edited the paper. 


\section{References}

Braver, T.S., Barch, D.M., Keys, B.A., Carter, C.S., Cohen, J.D., Kaye, J.A., Janowsky, J.S., Taylor, S.F., Yesavage, J.A., \&\& Mumenthaler, M.S. (2001). Journal of Experimental Psychology: General,, 130(4), 746-763.

Breukelaar, I.A., Antees, C., Grieve, S.M., Foster, S.L., Gomes, L., Williams, L.M., \& Korgaonkar, M.S. (2017). Cognitive control network anatomy correlates with neurocognitive behavior: a longitudinal study. Human Brain Mapping, 38(2), 631-643.

Bürkner P (2017). brms: An R Package for Bayesian Multilevel Models Using Stan. Journal of Statistical Software, 80, 1-28.

Bürkner P (2018). Advanced Bayesian Multilevel Modeling with the R Package brms. The R Journal, 10, 395-411.

Cabeza, R., Albert, M., Belleville, S., Craik, F., Duarte, A., Grady, C., Lindenberger, U., Nyberg, L., Park, D., Reuter-Lorenz, P.A., Rugg, M., Steffener, J., \& Rajah, M.N. (2018). Nature Reviews Neuroscience, 19(11), 701-710.

Cabeza, R., Anderson, N.D., Locantore, J.K., McIntosh, A.R. (2002). Aging gracefully: Compensatory brain activity in high-performing older adults. NeuroImage, 17, 13941402.

Cabeza, R. (2002). Hemispheric asymmetry reduction in older adults: The HAROLD model. Psychology and Aging, 17, 85-100.

Cabeza, R., Daselaar, S.M., Dolcos, F., Prince, S.E., Budde, M., \& Nyberg, L. (2004). Taskindependent and task-specific age effects on brain activity during working memory, visual attention, and episodic retrieval. Cerebral Cortex, 14, 364-375. 
Cappell, K.A., Gmeindl, L., \& Reuter-Lorenz, P.A. (2010). Age differences in prefrontal recruitment during verbal working memory maintenance depend on memory load. Cortex, 46, 462-473.

Chiu, Y. C., Lin, C. H., Huang, J. T., Lin, S., Lee, P. L., \& Hsieh, J. C. (2008). Immediate gain is long-term loss: Are there foresighted decision makers in the Iowa Gambling Task?. Behavioral and Brain Functions, 4, 13.

Daw, N. D., O’Doherty, J. P., Dayan, P., Seymour, B. \& Dolan, R. J. (2006). Cortical substrates for exploratory decisions in humans. Nature, 441, 876-879.

D'Esposito, M., Postle, B. R., Ballard, D., \& Lease, J. (1999). Maintenance versus manipulation of information held in working memory: an event-related fMRI study. Brain and cognition, 41, 66-86.

Don, H. J., Otto, A. R., Cornwall, A. C., Davis, T., \& Worthy, D. A. (2019). Learning reward frequency over reward probability: A tale of two learning rules. Cognition, 193, 104042.

Einhorn, H.J., \& Hogarth, R.M. (1981). Behavioral decision theory: Processes of judgment and choice. Annual Review of Psychology, 32, 53-88.

Elliot, R., Dolan, R.J., \& Frith, C.D. (2000). Dissociable functions in the medial and lateral orbitofrontal cortex: Evidence from human neuroimaging studies. Cerebral Cortex, 10, $308-317$.

Eppinger, B., Hammerer, D., \& Li, S.C. (2011). Neuromodulation of reward-based learning and decisionmaking in human aging. Annals of the New York Academy of Sciences, 1235, 117. 
1 Esteban, O., Markiewicz, C. J., Blair, R. W., Moodie, C. A., Isik, A. I., Erramuzpe, A., ... \& Oya, H. (2019). fMRIPrep: a robust preprocessing pipeline for functional MRI. Nature methods, 16, 111-116.

Estes, W.K. (1976). Some functions of memory in probability learning and choice behavior. In G.H. Bower (Ed.), The Psychology of Learning and Motivation (Vol. 10). New York: Academic Press.

Hare, T.A., O’Doherty, J., Camerer, C.F., Schultz, W., \& Rangel, A. (2008). Dissociating the role of the orbitofrontal cortex and the striatum in the computation of goal values and prediction errors. The Journal of Neuroscience, 28, 5623-5630.

Hillary, F. G., Genova, H. M., Chiaravalloti, N. D., Rypma, B., \& DeLuca, J. (2006). Prefrontal modulation of working memory performance in brain injury and disease. Human brain mapping, 27, 837-847.

Karayanidis, F., Whitson, L. R., Heathcote, A., \& Michie, P. T. (2011). Variability in proactive and reactive cognitive control processes across the adult lifespan. Frontiers in psychology, 2, 318 .

Li, S. C., Biele G., Lindenberger, U., \& Sikstrom, S. (2001). Aging cognition: from neuromodulation to representation. Trends in Cognitive Sciences, 5, 97-111.

Mather, M. (2006). A review of decision making processes: Weighing the risks and benefits of aging. In L.L. Carstensen \& C.R. Hartel (Eds.), When I'm 64: Committee on aging frontiers in social psychology, personality, and adult developmental psychology (pp. 145173). Washington, DC: National Academic Press. 
Mell, T., Heekeren, H.R., Marschner, A., Wartenburger, I., Villringer, A., \& Reischies, F.M. (2005). Effects of aging on stimulus-reward association learning. Neuropsychologia, 43, $554-563$.

Mohr, P.N.C., Li, S.C., \& Heekeren, H.R. (2010). Neuroeconomics and aging: Neuromodulation of economic decision-making in old age. Neuroscience and Biobehavioral Reviews, 34, 678-688.

Pagnoni G., Zink C. F., Montague P. R., and Berns G. S. (2002). Activity in the human ventral striatum locked to errors of reward prediction. Nature Neuroscience, 5, 97-98.

Park, D.C., \& Reuter-Lorenz, P. (2009). The adapting brain: aging and neurocognitive scaffolding. Annual Review of Psychology, 60, 173-196.

Pessiglione, M., Seymour, B., Flandin, G., Dolan, R. J., and Frith, C. D. (2006). Dopaminedependent prediction errors underpin reward seeking behavior in humans. Nature, 442, $1042-1045$.

Phillips, L.H., \& Andres, P. (2010). The cognitive neuroscience of aging: New findings on compensation and connectivity. Cortex, 46, 421-424.

Peters, E., Hess, T.M., Vastfjall, D., \& Auman, C. (2007). Adult age differences in dual information processes: Implications for the role of affective and deliberative processes in older adults' decision making. Perspectives on Psychological Science, 2, 1-23.

Raz, N. (2000). Aging of the brain and its impact on cognitive performance: integration of structural and functional findings. In The Handbook of Aging and Cognition, ed. F. Craik, T.A. Satlhouse, pp. 1-90. Hilsdale, NJ: Erlbaum. 
Raz. N., Linberger, U., Rodrique, K.M., Kennedy, K.M., Head, D., Williamson, A., Dahle, C., Gerstorf, D., \& Acker, J.D. (2005). Regional brain differences in aging healthy adults: General trends, individual differences and modifiers. Cerebral Cortex, 15, 1676-1689.

Rescorla, R.A., \& Wagner, A.R. (1972). A theory of Pavlovian conditioning: Variations in the effectiveness of reinforcement and non-reinforcement. In A.H. Black \& W.F. Prokasy (Eds.) Classical conditioning II: Current research and theory. New York: AppletonCentury-Crofts.

Resnick, S.M., Lamar, M., \& Driscoll, I. (2007). Vulnerability of the orbitofrontal cortex to ageassociated structural and function brain changes. Annals of the New York Academy of Sciences, 1121, 562-575.

Reuter-Lorenz, P.A., \& Cappell, K.A. (2008). Neurocognitive aging and the compensation hypothesis. Current Directions in Psychological Science, 17, 177-182.

Reuter-Lorenz, P.A., Jonides, J., Smith, E., Hartley, A., Miller, A., Marshuetz, C., \& Keoppe, R. (2000). Age differences in the frontal lateralization of verbal and spatial working memory revealed by PET. Journal of Cognitive Neuroscience, 12, 174-187.

Rodriguez, P.F., Aron, A.F., \& Poldrack, R.A. (2006). Ventral-striatal/nucleus-accumbens sensitivity to prediction errors during learning. Human Brain Mapping, 27, 306-313.

Samanez-Larkin, G.R., Wagner, A.D., \& Knutson, B. (2011). Expected value information improves financial risk taking across the adult life span. SCAN, 6, 207-217.

Schott, B.H., Niehaus, L., Wittman, B.C., Schutze, H., Seidenbecher, C.I., Heinze, H.J., \& Duzel, E. (2007). Ageing and early-stage Parkinson's disease affect separable neural mechanisms of mesolimbic reward processing. Brain, 130, 2412-2424. 
Sharp, D.J., Scott, S.K., Mehta, M.A., \& Wise, R.J. (2006). The neural correlating of declining performance with age: Evidence for age-related changes in cognitive control. Cerebral Cortex, 16(12), 1739-1749.

Stern, Y. et al. (2019). Mechanisms underlying resilience in ageing. Nature Review Neuroscience, 20, 246.

Widrow, B., \& Hoff, M.E. (1960). Adaptive switching circuits. 1960 WESCON Convention Record Part IV, 96-104.

Williams, R.J. (1992). Simple statistical gradient-following algorithms for connectionist reinforcement learning. Machine Learning, 8, 229-256.

Worthy, D. A., Davis, T., Gorlick, M. A., Cooper, J. A., Bakkour, A., Mumford, J. A., Poldrack, R. A., \& Maddox, W. T. (2016). Neural correlates of state-based decision-making in younger and older adults. Neuroimage, 130, 13-23.

Worthy, D.A., Gorlick, M.A., Pacheco, J.L., Schnyer, D.M., \& Maddox, W.T. (2011). With age comes wisdom: Decision-making in younger and older adults. Psychological Science, 22, 1375-1380.

Worthy, D. A., \& Maddox, W. T. (2012). Age-based differences in strategy use in choice tasks. Frontiers in neuroscience, 5, 145.

Worthy, D. A., Otto, A. R., Doll, B. B., Byrne, K. A., \& Maddox, W. T. (2015). Older adults are highly responsive to recent events during decision-making. Decision, 2, 27.

Worthy, D. A., Otto, A. R., Cornwall, A. C., Don, H. J., \& David, T., (2018). A case of divergent predictions made by delta and decay rule learning models. In T. T. Rogers, M. Rai, X. 
1 Yechiam, E., \& Ert, E. (2007). Evaluating the reliance on past choices in adaptive learning models. Journal of Mathematical Psychology, 51, 75-84.

3 Zhu, \& C. W. Kalish (Eds.) Proceedings of the $40^{\text {th }}$ Annual Conference of the Cognitive Science 4 Society (pp.1175-1180). Madison, Wisconsin: Cognitive Science Society. 